\title{
MODELO DE AVALIAÇÃO DA EXPOSIÇÃO E CARACTERIZAÇÃO DE RISCO À SAÚDE E MEIO AMBIENTE APLICADO A ATIVIDADE DE MINERAÇÃO.
}

\author{
MODEL OF EXPOSURE EVALUATION AND CHARACTERIZATION OF RISK TO HEALTH \\ AND ENVIRONMENT APPLIED TO MINING ACTIVITY.
}

\section{Sergio Fred Ribeiro ANDRADE, Fermin Garcia VELASCO, Francisco Heriberto Martinez LUZARDO, Jordan Brizi NERIS}

Universidade Estadual de Santa Cruz, Ilhéus - BA, Brasil. Emails: sergio.fred@hotmail.com; fermingv@gmail.com; fmartinezluzardo@gmail.com; jordanbrizi@gmail.com.

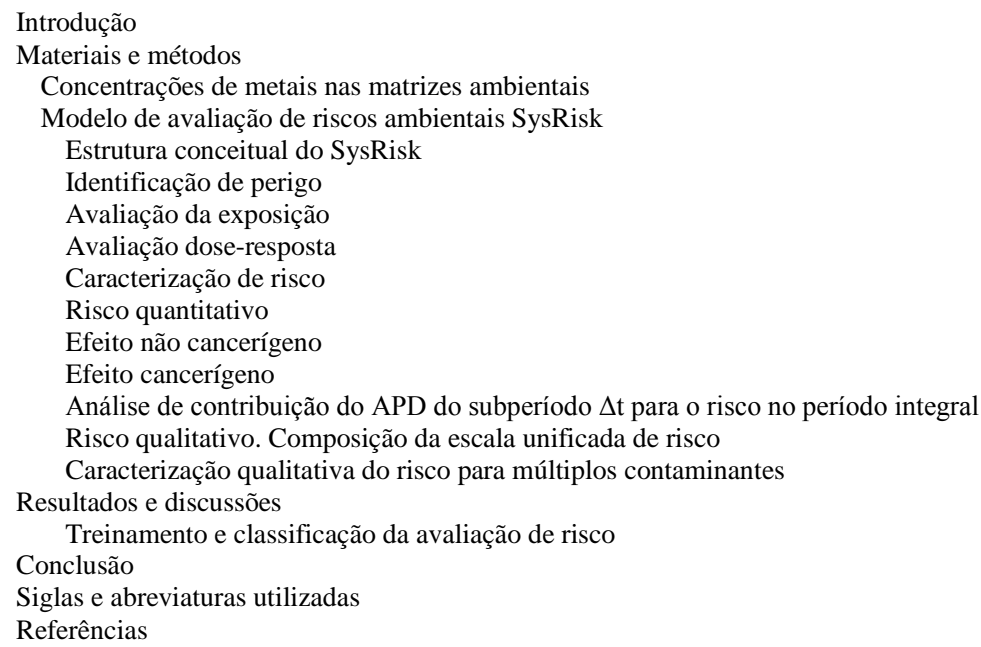

RESUMO - As metodologias para avaliação de risco à saúde são importantes ferramentas para auxiliar na gestão e mitigação de locais contaminados por compostos químicos. No presente trabalho, é proposta uma nova metodologia para avaliação de risco por exposição a compostos químicos, denominada SysRisk, que visa a avaliação dinâmica da exposição e caracterização do risco nas abordagens quantitativa e qualitativa, considerando a matriz espaço-temporal do cenário contaminado. O SysRisk foi aplicado em um cenário reportado por outros autores sobre a contaminação ambiental com metais $\mathrm{Fe}$, Zn, $\mathrm{Co}$ e $\mathrm{Pb}$, nos compartimentos ambientais água e solo, em área afetada pela atividade de uma metalúrgica de Zinco em Duque de Caxias, Rio de Janeiro - Brasil. Os resultados apurados pelo SysRisk foram comparados com os resultados do estudo reportado. A comparação mostrou diferenças significativas na caracterização do risco quantitativo e qualitativo entre as duas abordagens. O SysRisk mostrou adicionalmente a capacidade de realização de uma análise espaço-temporal detalhada que considera os efeitos individuais e sinérgicos de múltiplos contaminantes, e oferece novos subsídios para ações de mitigação mais eficientes diferenciadas por local afetado e período de tempo.

Palavras-chave: avaliação de risco; contaminação por metais; risco à saúde humana; mineração; Máquina Vetorial de Suporte.

\begin{abstract}
The methodologies for health risk assessment are important tools to assist in the management and mitigation of places contaminated by chemicals compounds. In this paper, it is proposed a new methodology for risk assessment by exposure to chemical compounds, called SysRisk, which aims the dynamic exposure assessment and risk characterization in quantitative approaches and qualitative approaches, considering the space-time matrix of the contaminated scenario. The SysRisk was applied in a scenario reported by other authors about the environmental contamination with metals $\mathrm{Fe}, \mathrm{Zn}, \mathrm{Co}$ and $\mathrm{Pb}$ in environmental compartments water and soil, caused by the activity of a metallurgical zinc in Duque de Caxias, Rio de Janeiro - Brazil. The results obtained by SysRisk were compared with the results of the reported study. The comparison showed significant differences in the characterization of quantitative and qualitative risk between the two approaches. The SysRisk additionally showed the ability to carry out a detailed spatio-temporal analysis that considers the individual and synergistic effects of multiple contaminants, and offers new subsidies for more efficient mitigation actions differentiated by affected place and time period.
\end{abstract}

Keywords: Risk assessment; metal contamination; risk to human health; mining; Support Vector Machine.

\section{INTRODUÇÃO}

A avaliação de risco é uma ferramenta importante para a busca de soluções em problemas de exposição por contaminantes químicos, e na gestão do risco e remediação de áreas contaminadas. Esta ferramenta está ligada à exigência de novos regulamentos ambientais, como se destaca em diversos trabalhos recentes, como os de Guo et al. (2013) e Cao et al.
(2011).

Da mesma forma, Linkov et al. (2009) e Dourson et al. (2001) apontam que as decisões na gestão do risco dependem fundamentalmente da avaliação do risco em base científica, que auxiliam tanto o setor privado quanto as autoridades públicas. Esta questão é também destacada em Brilhante \& Caldas (2004) e 
Souza (2012), que enfatizam a atualidade e importância do tema, e, em particular, a potencialidade da utilização dessa ferramenta para auxiliar os sistemas de gestão ambiental do setor produtivo.

Nos Estados Unidos, União Europeia e em alguns países, as metodologias de avaliação de risco por produtos nocivos à saúde humana são apoiadas por legislação exigente que inclui a aplicação de procedimentos bem definidos para auxiliar na eliminação das fontes emissoras de resíduos perigosos. Mesmo assim, como é apontado em várias publicações como WHO (2010) e USEPA (2000), ainda são metodologias, que devem ser aprimoradas especialmente no que concerne à comunicação direta para à sociedade.

As principais metodologias para avaliação de risco à saúde são as disponíveis pela Agência de Proteção Ambiental dos Estados Unidos - USEPA (1989, 2004a, 2004b), Organização Mundial da Saúde - WHO (2010), União Europeia - UE (2003) e pelos governos do Canadá (HEALTH CANADA, 2004) e Holanda (RIVM, 2009).

No Brasil, a Companhia Ambiental do Estado de São Paulo - CETESB (2003) tem publicado procedimentos importantes para o processo de avaliação do risco à saúde humana. Esses métodos, de forma geral, tem uma abordagem estritamente quantitativa com atributos e padrões numéricos. Estes atributos carregam algumas vantagens relacionadas com o fato de facilitarem a medição e comparação de cenários e determinação de limites seguros.

Por outro lado, nessas metodologias o risco é caracterizado apenas com base em limites quantitativos, que às vezes podem reduzir a informação a um aparente sistema binário de avaliação, com resultado "existe risco; não existe risco”. Essa situação pode limitar a avaliação dos diferentes graus de riscos, assim como a compreensão correta das diferenças qualitativas dos tipos de riscos e a eficiência na sua comunicação à sociedade.

Alguns autores, Marhavilas et al. (2011) e Menzie \& Freshman (1997), levantam essa questão e apontam que a maioria das metodologias de avaliação de risco à saúde humana e ao ambiente apresentam abordagem somente quantitativa, deixando sem caracterização qualitativa os riscos avaliados a que a população está exposta.

O Governo Brasileiro, através da FUNDACENTRO (2013), menciona a ênfase exagerada no critério quantitativo para avaliação do risco à saúde humana, possibilitando bloqueios de ações preventivas devido à incapacidade de interpretação dos números. De acordo com Barnes et al. (1988) e Renwick et al. (2003), a avaliação de risco com referencial toxicológico de exposição privilegia uma dose limiar, sem aproximar a avaliação para uma indicação qualitativa dos efeitos deletérios que possam ser provocados.

No trabalho de Edler et al. (2002), é feita uma ampla revisão dos modelos quantitativos aplicados na caracterização de risco, onde são apontadas deficiências e desvantagens dos métodos baseados unicamente no limite referencial quantitativo como o ADI (Dose de Ingestão Aceitável), TDI (Dose Diária Tolerável), RfD (Dose de Referência), NOAEL (Nenhum Nível Efeito Adverso Observado).

Os autores argumentam que nestes métodos não são caracterizados claramente os diferentes níveis dos efeitos adversos gerados pelo contaminante, além de incluir ainda um elevado grau de incertezas nos estudos.

No trabalho reportado por Lima (2009), foi realizada uma avaliação dos possíveis impactos na saúde e meio ambiente derivados da contaminação por metais tóxicos em uma área de uma empresa metalúrgica de zinco desativada.

O trabalho de tese de Lima objetivou auxiliar no processo de descomissionamento da empresa visando elaborar as medidas necessárias a serem tomadas para mitigar os riscos provocados. A autora utilizou uma abordagem toxicológica baseada nas metodologias de avaliação de risco à saúde humana e ecológico propostas pela USEPA (1989) (United States Environmental Protection Agency), bem como a realização de testes ecotoxicológicos com minhocas (Eisenia foetida).

Foram realizadas medidas experimentais das concentrações de metais em solo e água em pontos de coleta da área afetada, sendo estes valores tratados como concentrações de exposição ambiental constantes no tempo. A partir destes dados, utilizando os modelos e parâmetros reportados na literatura foram 
estimados os teores de metais transportados até os receptores humanos e ecológicos em diferentes cenários hipotéticos de uso futuro da área. Para todos os cenários estudados a autora obteve valores de riscos considerados inaceitáveis. Na avaliação de risco à saúde humana, os maiores níveis de risco foram para $\mathrm{Cd}, \mathrm{Pb}, \mathrm{Hg}$ e $\mathrm{Zn}$, no cenário agrícola para crianças expostas via ingestão e contato dérmico com solo e água subterrânea.

$\mathrm{Na}$ avaliação de risco ecológico, os maiores níveis de risco foram para invertebrados, microrganismos, pequenos mamíferos e plantas, para Al, Cd, Fe, Hg, Se e Zn. Face aos resultados da análise de risco ambiental, a autora sugere uma intervenção na área.

Lima (2009), apresenta uma metodologia quantitativa baseada em estudos toxicológicos biológicos que são apresentados apenas em estimativas numéricas ou índices como o Hazard Index para metais não cancerígenos e o Risco Cancerígeno para substâncias com potencial cancerígeno, sem indicação sobre efeitos adversos à saúde e categorização do risco avaliado.

Por outro lado, esses métodos, em geral, utilizam uma aproximação na avaliação da exposição que consideram a concentração do contaminante constante (com seu valor médio), durante longos períodos de exposição. Esse tipo de abordagem facilita os cálculos e comparação de cenários, mas impede uma análise mais cuidadosa em períodos de exposição mais curtos, de exposição aguda, como, por exemplo, em acidentes.

A utilização nos cálculos de exposição, da concentração média no lugar da função temporal real da concentração do contaminante, conduz a um conhecimento pouco rigoroso da evolução temporal da situação de risco. Esse fato pode prejudicar a efetividade das ações de mitigação da área afetada em intervalos intermediários.

A partir das considerações mencionadas, e com base nos dados apresentados por Lima (2009) o objetivo deste trabalho foi propor um novo modelo de avaliação de risco SysRisk, que incluiu, inicialmente, uma abordagem quantitativa, por índice de perigo e risco cancerígeno, mais detalhado e específico para cada período temporal e espaço amostral do cenário estudado, e que considerou a exposição com base na função temporal da concentração dos contaminantes. A seguir, foi desenvolvida uma nova metodologia com abordagem qualitativa que utiliza como base a categorização ordinal do risco.

\section{MATERIAIS E MÉTODOS}

\section{Concentrações de Metais nas Matrizes Ambientais}

A metodologia SysRisk proposta neste trabalho, foi aplicada com base nos dados da avaliação da exposição de risco à saúde humana, reportados por Lima (2009), que tratam sobre o descomissionamento de uma metalúrgica de beneficiamento de minério de zinco e seus metais associados, localizada em Duque de Caxias, Rio de Janeiro - Brasil. A metalúrgica localiza-se próxima ao Rio Calombé, que até o presente momento recebe efluente advindo de água subterrânea contaminada.

O presente trabalho não pretendeu refazer uma análise completa do caso desta metalúrgica, utilizando o conjunto completo de dados de concentrações ambientais com todos os metais e rotas de exposição. Uma parte dos dados foi utilizada visando apenas ilustrar os resultados com a metodologia proposta SysRisk; e comparando-os com os resultados obtidos por Lima (2009), no intuito de discutir as diferenças de ordem quantitativa e qualitativa entre os métodos.

Foram selecionados, para o presente estudo, os dados das concentrações dos metais Fe, Zn, Co e $\mathrm{Pb}$ para água subterrânea e solo, em três pontos amostrais distantes entre si, denominados A1, A2 e A3 no plano de amostragem original de Lima (2009).

As concentrações das amostras de água e solo, reportadas por Lima (2009), foram analisadas pelos autores usando a técnica de espectrometria de absorção atômica com chama, para amostras do ano de 2005.

Visando considerar a situação real típica de descomissionamento da mineradora sob estudo, e mostrar a possibilidade de análise temporal pelo SysRisk, foram simuladas as funções temporais das concentrações dos contaminantes em água e solo durante os 24 anos considerados no cenário residencial para adultos por CETESB (2001).

O cálculo de Lima (2009) adotou o valor medido das concentrações como constante em 
todo período de 24 anos.

Na simulação realizada no presente trabalho, foi considerado um cenário com mitigação, em que os valores das concentrações dos contaminantes são reduzidos gradualmente, de dois em dois anos, até alcançar o valor limite permitido (CETESB 2005) ao fim de um período de 10 anos.

Dentro de cada período de 2 anos, considerouse as concentrações constantes. Durante o período residual de 14 anos, considerou-se as concentrações dos contaminantes fixadas nos limites permitidos.

Desta forma, a função temporal da concentração foi considerada aplicando a equação 1:

$$
\mathrm{C}\left(\mathrm{t}_{\mathrm{i}}\right)=\mathrm{C}\left(\mathrm{t}_{\mathrm{i}-1}\right)-\Delta \mathrm{C}
$$

Onde:

$$
\Delta \mathrm{C}=\left(\mathrm{C}_{\max }-\mathrm{C}_{\mathrm{R}}\right) / \mathrm{n}
$$

Onde $\Delta \mathrm{C}$ é o fator de redução da concentração; $\mathrm{C}_{\text {max }}$ é a concentração em $\mathrm{mg} \mathrm{kg}^{-1}$ reportada por Lima (2009); $C_{R}$ é valor de referência em CETESB (2005) para o metal específico; $n$ representa o número de períodos em que foi subdividido o período total de tempo de 24 anos, neste caso 5 ; $\mathrm{C}\left(\mathrm{t}_{\mathrm{i}}\right)$ representa a concentração do período atual a ser estimado; e $\mathrm{C}\left(\mathrm{t}_{\mathrm{i}-1}\right)$ é a concentração no período anterior.

\section{Modelo de Avaliação de Riscos Ambientais Sysrisk}

O SysRisk faz uma adaptação da metodologia empregada pela USEPA (1989), visando oferecer recursos na interpretação qualitativa do risco à saúde humana, no sentido de caracterizar melhor a classificação do risco em categorias ordinais.

A nova metodologia SysRisk propõe:

a) uma complementaridade na formulação geral da avaliação da exposição para análises temporais pela evolução da duração e frequência da exposição por contaminante;

b) incluir uma escala unificada de risco com níveis categorizados de risco baseados nos indicadores do índice de perigo, risco cancerígeno, grupos de carcinogênicos, NOAEL/LOAEL (Nível Baixo de Efeito Adverso Observado) e dose de letalidade;

c) a utilização da técnica matemáticocomputacional de máquinas de suporte vetoriais para determinar a classificação do risco. Estes aspectos serão detalhados a seguir.

\section{Estrutura Conceitual do SysRisk}

A estrutura conceitual para aplicação do SysRisk é baseada no roteiro da NRC (1983), que inclui a identificação do perigo, a avaliação da exposição, avaliação dose-resposta e caracterização do risco como etapas básicas para a avaliação do risco à saúde humana (Figura 1).

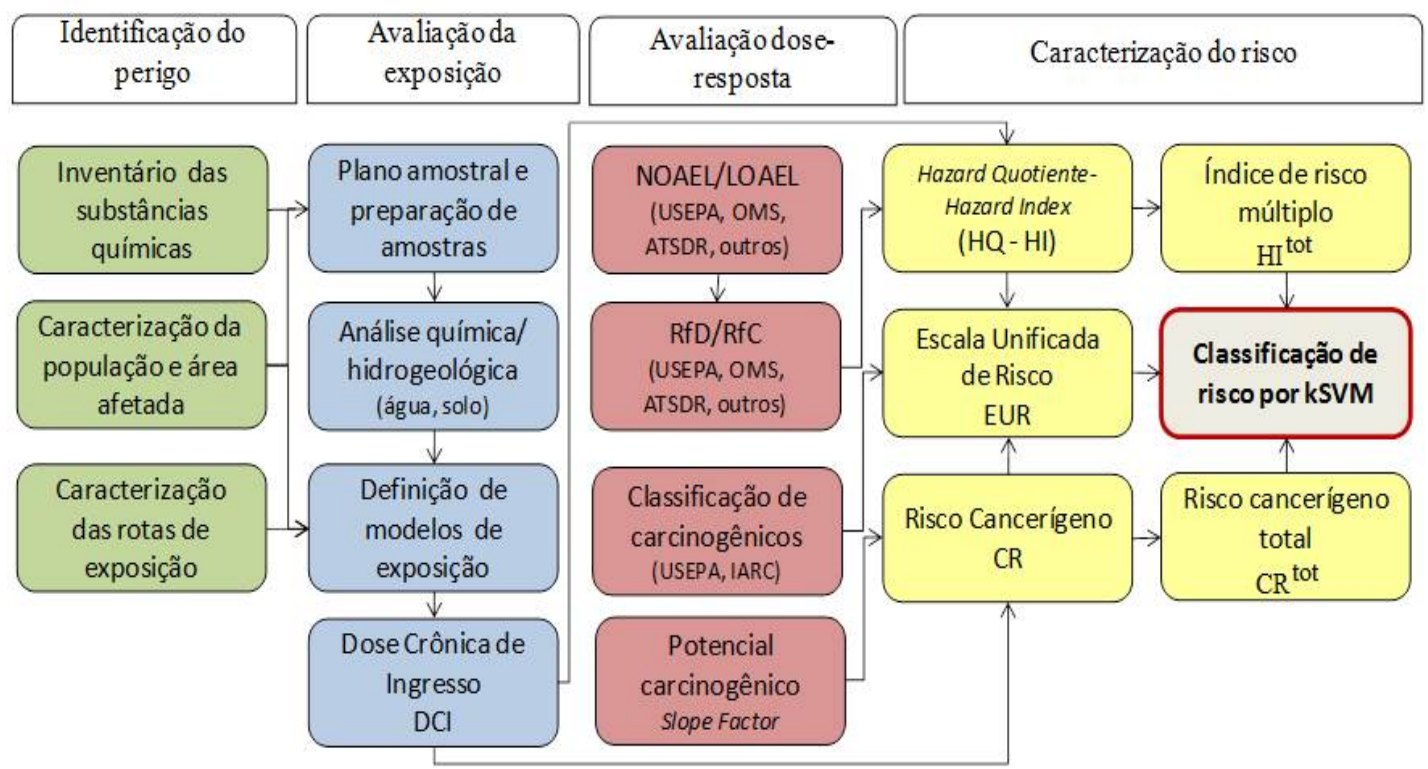

Figura 1 - Esquema da metodologia do SysRisk de Avaliação de Risco.

\section{Identificação do Perigo}

Na etapa da identificação do perigo é necessária a descrição dos elementos contaminantes presentes no meio ambiente. São identificadas as substâncias químicas de interesse, o potencial de toxicidade, as concentrações ambientais, as rotas de migração, o transporte, destino e persistência dos contaminantes. São 
caracterizadas também as propriedades geoquímica, física, de clima e da biota do local afetado e é realizada a descrição da atividade, do evento gerador da contaminação, da população e dos grupos sensíveis receptores da exposição dos compostos químicos.

\section{Avaliação da Exposição}

Foi considerado, como base, o modelo geral de avaliação da exposição, conforme formulação básica de USEPA (1992) para definição das equações para cálculo da dose potencial, de acordo com a equação 2 .

$$
D_{p o t}=\int_{t_{1}}^{t_{2}} C(t) I R(t) d t
$$

Onde: $D_{\text {pot }}$ é a dose potencial (mg dia $\left.{ }^{-1}\right)$; $\mathrm{C}(\mathrm{t})$ é a concentração da exposição em função do tempo (mg g ${ }^{-1}$, mg L $\left.{ }^{-1}\right) ; t_{2}-t_{1}$ é a duração do tempo de exposição avaliado; e IR(t) é a taxa de ingestão, inalação ou contato dérmico do contaminante (g dia ${ }^{-1}, \mathrm{~L} \mathrm{dia}^{-1}$ ); que depende da natureza deste último e da via de ingresso.

A equação 3 constitui a expressão geral da dose, considerando a concentração do contaminante uma função do tempo. A utilização dessa equação impõe a necessidade de conhecer a função temporal da concentração o mais detalhadamente possível, aproximadamente por dia, em períodos de exposição que podem estender-se por décadas. Por isso, na prática, em muitos estudos que aplicam as metodologias de risco, USEPA (2004), OMS (2010), PNUMA (1999) e UE-IHCP (2003), no lugar da equação 3 adota-se a função temporal da concentração como uma constante, que pode expressar o maior valor dessa concentração no período avaliado ou seu valor médio, sendo essa equação 3 expressa na aproximação da equação 4 para determinação da dose média diária.

$$
\mathrm{ADD}=\frac{\mathrm{C} \times \mathrm{ED} \times \mathrm{EF}}{\mathrm{BW} \times \mathrm{AT}}(\mathrm{IR})
$$

Onde: ADD é a dose potencial média de ingresso do contaminante no período de tempo de exposição em mg kg-1 dia-1; C é a concentração média no período da exposição em mg kg-1, mL L-1, ou mg $\mathrm{cm}^{-3}$; ED é a duração do tempo da exposição em anos; EF é a frequência média de exposição em dias ano-1; IR é a taxa de ingestão, inalação ou contato dérmico por dia em cm3 dia-1, kg dia-1, L dia1; BW é o peso corpóreo médio da população em kg; e AT é o tempo total em dias de vida dos receptores. Sendo a constante C a concentração média no período, o resultado conduz a valores médios da dose e risco, considerando todo o período avaliado.

Esse valor médio $C$ da concentração pode mascarar períodos alternados de valores altos e baixos da exposição. Portanto, podem ser camuflados possíveis riscos maiores pela exposição naqueles períodos de concentrações mais altas. Por outro lado, caso se utilize a maior concentração registrada no período, com um valor constante para $C$ da equação 4 , é possível fazer um cálculo conservador onde os níveis de riscos nunca serão subestimados. Porém, nessa abordagem, não é possível considerar os níveis de concentrações variáveis que possam indicar a realidade de picos de exposições e elevações no seu limiar, devido a acidentes ambientais, elevação dos índices pluviométricos, ou aumento ou diminuição na frequência da exposição por receptores.

No presente trabalho não são utilizadas as concentrações constantes em períodos longos, como aparece na formulação da equação 3, assim como não é utilizada a função temporal da concentração com a variável temporal contínua, como ocorre na formulação da equação 4. A concentração será considerada constante apenas dentro do período $\Delta t$, o qual pode ser, por exemplo, o tempo entre coletas de amostras utilizadas para determinação experimental da concentração dos contaminantes nas diversas matrizes ambientais. Desta forma, será utilizada uma abordagem discreta para a variável temporal, expressa nas equações 5 e 6 a seguir.

$$
\begin{aligned}
& \operatorname{PD}\left(t_{i}\right)=\mathrm{C}_{\exp }\left(\mathrm{t}_{\mathrm{i}}\right) \cdot(\Delta \mathrm{t}) \cdot \mathrm{EF}_{\mathrm{f}}\left(\mathrm{t}_{\mathrm{i}}\right) \cdot \operatorname{IR}_{\mathrm{j}}\left(t_{i}\right) \\
& \text { para } \Delta \mathrm{t}=\mathrm{t}_{\mathrm{i}}-\mathrm{t}_{\mathrm{i}-1} \text { e } \mathrm{i}=(1,2, \mathrm{n}) \\
& \mathrm{e}
\end{aligned}
$$

$$
\operatorname{APD}\left(t_{i}\right)=\frac{\mathrm{PD}\left(t_{i}\right)}{\overline{\mathrm{BW}} \times \mathrm{AT}}
$$

Onde: $\operatorname{PD}\left(t_{i}\right)$ é a Dose parcial de Ingresso no período de tempo $\Delta t$ através de uma rota de exposição, em mg kg $\mathrm{kia}^{-1} ; C_{\text {exp }}\left(\mathrm{t}_{\mathrm{i}}\right)$ é a concentração em mg kg-1 $\mathrm{mL} \mathrm{L}^{-1}$, ou mg cm ${ }^{-3}$ na rota de exposição para um período de tempo determinado $\Delta t$, que é a duração da exposição por período amostral (em dia), onde $t_{i}$ é o tempo final do período intermediário e $t_{i-1}$ é o tempo inicial do mesmo período; $E F_{f}\left(t_{i}\right)$ é a frequência 
variável da exposição para o período intermediário $t_{i}$, expressa como uma fração adimensional do $\Delta t$. Assim, o produto $\left(\Delta \mathrm{t}_{\mathrm{EF}}\right)$ expressa o número efetivo de tempo (em dias) da exposição.

A variável $\operatorname{IR}_{\mathrm{j}}\left(t_{i}\right)$ representa os parâmetros de ingresso específicos por rota de exposição e contaminante ou conversores de medidas. A grandeza $\operatorname{APD}\left(t_{i}\right)$ representa a média pelo período integral de exposição AT e kg de peso da dose recebida no período $\Delta t$, que expressa o valor com que cada fração temporal contribui para a dose média por dia e $\mathrm{kg}$ de peso, acumulada durante o período total de exposição.

Visando fazer a análise dependente do tempo, introduzimos na equação 6 a grandeza $A D_{a c}\left(t_{j}\right)$ como a dose média acumulada até o período $\Delta \mathrm{t}=$ $\left(t_{i+1}-t_{i}\right)$, incluindo este, expresso na equação 7 , que é calculada como a média no período em que ocorre a exposição e por kg de peso corpóreo.

$$
\operatorname{AD}_{\mathrm{ac}}\left(\mathrm{t}_{\mathrm{j}}\right)=\frac{1}{\overline{\mathrm{BW}} \mathrm{xAT}} \cdot \sum_{i=1}^{j} \mathrm{PD}\left(t_{i}\right)
$$

Onde o parâmetro $\overline{\mathrm{BW}}$ representa o peso médio corpóreo em kg na subpopulação afetada; e $A T$ é o tempo total em que acontece a exposição em dias.

Para calcular a dose acumulada total no período integral (como avaliada na equação 3, é preciso fazer a soma da contribuição de todos os tempos parciais na dose total, conforme equação 8.

$$
A D D^{*}\left(\mathrm{t}_{\mathrm{j}}\right)=\frac{1}{\overline{\mathrm{BW} \times \mathrm{AT}}} \cdot \sum_{i=1}^{f} \operatorname{PD}\left(t_{i}\right)
$$

Onde: $\mathrm{ADD}^{*}$ é a dose de ingresso do contaminante acumulada no período de tempo total em mg kg-1 $\mathrm{dia}^{-1}$; e o índice $i$ representa $o$ número total $f$ de períodos em que foi subdividido o tempo de exposição total.

A diferença básica entre ADD e ADD consiste apenas no nível de detalhamento temporal dos fatores da exposição.

A utilização das equações 5 e 6 , no lugar da equação 4, vai possibilitar a avaliação dinâmica da exposição por período sazonal e permite caracterizar o risco a partir da evolução real das concentrações dos contaminantes, da frequência e do tipo de exposição ao contaminante, considerando, de forma mais realista, as mudanças de cenários ambientais e produtivos de atividades econômicas poluentes de longo prazo, como a mineração e metalurgia.
Para o caso de exposição às substâncias cancerígenas, é calculado o LADD ${ }^{*}, \mathrm{LAD}_{\mathrm{ac}}$ com as mesmas fórmulas para $\mathrm{AD}_{\mathrm{ac}}$ e $\mathrm{ADD}^{*}$, equações 7 e 8 , respectivamente, considerando diferentemente apenas o AT que, para o caso cancerígeno, será o tempo de vida médio da população.

Para avaliar as diferentes rotas foram adotadas as formulações e parâmetros com detalhes dos cenários em "Example Exposure Scenarios”, USEPA (2004), e dados dos fatores de exposição adotados no Brasil e publicados no CETESB (2001). Para cenários de exposição crônica, como os da atividade de mineração, a grandeza ADD pode ser denominada como DCI (Daily Chronic Intake).

\section{Avaliação Dose-Resposta}

As doses de referências da Avaliação DoseResposta para os contaminantes não carcinogênicos foram adotadas por rota de exposição, listadas em ordem de prioridade de acordo com a sua disponibilidade: RfD/RfC (Dose Referência/Concentração Referência) USEPA (1989), ADI (Dose de Ingestão Aceitável) ou TDI (Dose Diária Tolerável) WHO (2010), MRL (Mínimo Nível de Risco) ATSDR (2005), ACI (Dose Aceitável para Exposição Crônica) - USEPA (1989).

As doses referenciais foram selecionadas para efeito toxicológico crônico, mais adequadas à exposição derivadas da atividade de mineração.

$\mathrm{Na}$ avaliação da toxicidade das substâncias carcinogênicas é empregado o potencial cancerígeno (slope factor). Para as classificações toxicológicas carcinogênicas são usadas as tabelas de grupos por peso-da-evidência (weigthof-evidence), publicadas pela USEPA (2015) e IARC-WHO (2015).

\section{Caracterização de Risco}

De acordo a metodologia proposta SysRisk, o procedimento de caracterização de risco é subdividido nas seguintes etapas: 1) determinação do Índice de Perigo - Hazard Index (HI) e do Risco Cancerígeno (CR) por contaminante e por espaços e períodos amostrais; 2) aplicação da Escala Unificada de Risco (EUR), que inclui HI e CR nos cenários estudados; 3) classificação do risco categórico ordinal por Máquina de Suporte Vetorial para Multiclassificação (kSVM), Rifkin \& Klautau (2004) e Hsu \& Lin (2002), para caracterização qualitativa do risco, incluindo a 
determinação do risco simultâneo para múltiplos contaminantes por espaços e períodos amostrais.

\section{Risco Quantitativo}

Os procedimentos de cálculo do risco quantitativo seguem as formulações da USEPA (1989), conforme as seguintes etapas: a) para as substâncias não cancerígenas, são determinados o (HQ) Hazard Quotient - Quociente de Perigo para cada substância e rota de ingresso específica, o Î́ndice de Perigo (HI) por substância específica, considerando as várias rotas de ingresso e o Índice de Perigo Múltiplo $\left(\mathrm{HI}^{\mathrm{tot}}\right)$ para exposição simultânea a várias substâncias e rotas possíveis; e, b) para as substâncias cancerígenas são determinados o Risco Cancerígeno (CR), e de forma similar, os indicadores com soma de efeitos de várias rotas e substâncias simultâneas o Risco Cancerígeno Múltiplo ( $\mathrm{CR}^{\text {mult }}$ ) e o Risco Cancerígeno Total $\left(\mathrm{CR}_{\mathrm{tot}}\right)$.

\section{Efeitos não Cancerígenos}

Após a obtenção da $\mathrm{AD}_{\mathrm{ac}}(\mathrm{t})$ por contaminantes não cancerígenos, a etapa seguinte é a determinação do $\mathrm{HQ}(\mathrm{t})$ de um contaminante específico pela equação 9 e do Índice de perigo HI(t), utilizando a aproximação aditiva de exposição de todas as rotas pela equação 10 , USEPA (1989).

$$
\begin{aligned}
& \mathrm{HQ}_{\mathrm{i}}(\mathrm{t})=\mathrm{AD}_{\mathrm{ac}}^{\mathrm{i}}(\mathrm{t}) / \mathrm{RfD} \\
& \mathrm{HI}(\mathrm{t})=\sum_{\mathrm{i}=1}^{\mathrm{n}} \mathrm{HQ}_{\mathrm{i}}(\mathrm{t})
\end{aligned}
$$

Onde: HI(t) é o índice de perigo no tempo $t$ (com base na exposição acumulada até o tempo t) para exposição à múltiplas rotas; $A D_{a c}^{i}(t)$ é a dose obtida pelas equações 7 para a $i$-ésima rota de exposição até o tempo $t$, estimada em (mg $\mathrm{kg}^{-1} \mathrm{dia}^{-1}$ ); e RfD é a dose de referência ( $\mathrm{mg} \mathrm{kg}$ ${ }^{1} \mathrm{dia}^{-1}$ ) para a substância e rota considerada.

A ação simultânea da exposição a vários contaminantes segue a aproximação de efeito aditivo da USEPA (1989), e é calculada na equação 11 .

$$
\mathrm{HI}^{\mathrm{tot}}(\mathrm{t})=\sum_{\mathrm{j}=1}^{\mathrm{m}} \mathrm{HI}_{\mathrm{j}}(\mathrm{t})
$$

Onde $\mathrm{HI}^{\mathrm{tot}}(\mathrm{t})$ representa o índice de perigo para o evento contaminante num ponto da matriz espaço-temporal.

\section{Efeitos Cancerígenos}

Para cada rota de exposição a substâncias cancerígenas, é calculado o (CR) Risco Cancerígeno pela equação 12, para baixas doses de ingresso diárias ( $\mathrm{CR} \leq 0,01)$, conforme USEPA (1989).

$$
\mathrm{CR}(\mathrm{t})=\mathrm{LAD}_{\mathrm{ac}}(\mathrm{t}) \mathrm{x} \quad \mathrm{SF}
$$

(Slope Factor (fator potencial cancerígeno)

Em cenários com alta dose de ingresso diária (CR > 0,01), a equação (09) linear não é mais válida e o $C R$ pode ser estimado pela equação 13.

$$
\mathrm{CR}(\mathrm{t})=1-\exp \left(-\mathrm{LAD}_{\mathrm{ac}}(\mathrm{t}) \times \mathrm{SF}\right)
$$

Onde: CR é a probabilidade de um indivíduo desenvolver câncer ao longo da vida, considerando a exposição de contaminantes até o tempo $t$; SF é o fator potencial cancerígeno (slope factor) medido em (mg kg $\left.{ }^{-1} \mathrm{dia}^{-1}\right)$; e $\mathrm{LAD}_{\mathrm{ac}}(t)$ é a dose de ingresso diária calculada para o contaminante dado, considerando a exposição até o tempo $t$ por uma rota específica.

$\mathrm{Na}$ caracterização do Risco Cancerígeno Múltiplo $\left(\mathrm{CR}^{\text {mult }}\right)$, para múltiplas rotas, são somadas as probabilidades do $\mathrm{CR}$, conforme a equação 14.

$$
\mathrm{CR}^{\text {mult }}(\mathrm{t})=\sum_{\mathrm{i}=1}^{\mathrm{n}} \mathrm{CR}_{\mathrm{i}}(\mathrm{t}) 14
$$

Na equação 15) são consideradas as várias substâncias simultâneas (j), seguindo USEPA (1989).

$$
C R^{\text {tot }}(t)=\sum_{j=1}^{m} C R_{j}^{\text {mult }}(t)
$$

\section{Análise de Contribuição do APD do Subperíodo At para o Risco no Período Integral}

No intuito de conhecer a fração do risco que a dose de um subperíodo determinado acrescenta ao risco no tempo integral de exposição, foram realizados cálculos de $\mathrm{HI}$ e $\mathrm{CR}$ substituindo as grandezas aditivas $\mathrm{AD}_{\mathrm{ac}}(\mathrm{t})$ e $\mathrm{LAD}_{\mathrm{ac}}(\mathrm{t})$ pela correspondente a um subperíodo: $\operatorname{APD}(\mathrm{t})$ e LAPD(t), utilizando as grandezas parciais por subperíodo (sp):

$$
\begin{aligned}
& \mathrm{HQ}_{\mathrm{i}}(\mathrm{t})_{\text {sp }}=\mathrm{APD}^{\mathrm{i}}(\mathrm{t}) / \mathrm{RfD} \\
& \mathrm{CR}(\mathrm{t})_{\text {sp }}=\mathrm{LAPD}_{\text {pot }}(\mathrm{t}) \times \mathrm{SF}
\end{aligned}
$$

A partir das equações (16) e (17) serão obtidas as grandezas por subperíodo $\mathrm{HI}_{\mathrm{sp}}, \mathrm{HI}^{\text {tot }}{ }_{\mathrm{sp}}, \mathrm{CR}_{\mathrm{sp}}^{\text {mult }}$, $\mathrm{CR}^{\mathrm{tot}}$ sp.

\section{Risco Qualitativo. Composição da Escala Unificada de Risco}

A avaliação qualitativa de risco é o cerne da nova proposta do presente trabalho.

Nesta proposta, o risco qualitativo é avaliado 
por classes que representam categorias ordinais (de 1 a 5), conforme Escala Unificada de Risco (EUR) apresentada na tabela 1.

A EUR permite a atribuição de classes ordinais de efeitos adversos à saúde, de acordo com uma composição entre o HI e CR de USEPA (1989), os grupos carcinogênicos de USEPA (2015) e IARC (2015) e das doses/concentrações tóxicas letais WHO (2015). A aplicação da EUR justifica-se pela necessidade de categorização ordinal de risco para a fase de treinamento de dados no ambiente supervisionado por kSVM (Máquina Vetorial de Suporte para Multiclassificação), que será utilizada para classificação do risco na etapa final da caracterização qualitativa.

Tabela 1 - Escala Unificada de Risco. Adaptado de USEPA (2015), EPCRA-Section 304 (2014).

\begin{tabular}{|c|c|c|}
\hline Critério de categorização & Conceito & $\begin{array}{l}\text { Classe de } \\
\text { Risco }\end{array}$ \\
\hline $\mathrm{HI} \leq 1$ & Nenhum efeito & 1 \\
\hline HI > 1 e ADD* < LOAEL. & Efeito adverso & 2 \\
\hline $\begin{array}{l}\text { HI }>1 \text { e ADD* } \geq \text { LOAEL; ou RfD crônica }^{\text {a }} \text {; e ADD } *<\text { DL }_{50}\left(\text { DC }_{50}\right) \\
\text { ou Grupo B2 - USEPA } \text {; ou CR }<10^{-6} \text {. }\end{array}$ & Efeito irreversível $^{\mathrm{d}}$ & 3 \\
\hline 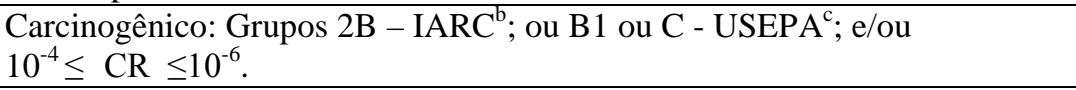 & Efeito grave & 4 \\
\hline $\begin{array}{l}\text { HI }>1 \text { e ADD* } \geq \text { LOAEL; ou RfD crônica }{ }^{\mathrm{a}} \text { e } \mathrm{ADD}^{*} \geq \mathrm{DL}_{50}\left(\mathrm{DC}_{50}\right) \\
\text { Cancerígeno: Grupos } 1 \text { ou } 2 \mathrm{~A} \text { - IARC } \text { IA }^{\mathrm{b}} \text { ou A ou B - USEPA } \text {; ou CR }>10^{-4} \text {. }\end{array}$ & Efeito gravíssimo & 5 \\
\hline
\end{tabular}

Os critérios para definição das diferentes categorias de risco na EUR proposta são descritos a seguir.

A categoria um (1) de risco corresponde ao caso em que HI é menor ou igual ao RfD, e corresponde à caracterização de risco onde nenhum efeito adverso à saúde humana foi observada.

A categoria dois (2) de risco corresponde a um HI superior a 1,0 e ADD* menor que a dose correspondente ao LOAEL (Baixo Nível de Efeito Adverso Observado), WHO (2010), onde foi observado e publicado oficialmente algum efeito adverso à saúde moderado, como perda de peso, irritabilidade ou outra adversidade moderada.

A categoria três (3) de risco corresponde a um $\mathrm{HI}$ superior a 1,0 e ADD* maior ou igual à dose correspondente ao LOAEL; ou ao nível de RfD crônico, onde foi reportado algum efeito adverso importante, como intoxicação crônica, irreversibilidade, disfunções reprodutivas, neurológicas ou em órgãos vitais, mutagenicidade ou outra adversidade congênere; sendo a $\mathrm{ADD}^{*}$ menor que $\mathrm{DL}_{50} / \mathrm{CL}_{50}$. $\mathrm{Ou}$ o contaminante seja classificado como B2 pela USEPA (2015) ou apresente Risco Cancerígeno (CR) menor que $10^{-6}$.

A categoria quatro (4) de risco corresponde ao enquadramento do contaminante como $2 \mathrm{~B}$ pela IARC (2015), ou B1 ou C da USEPA (2015) - considerada como efeito grave por relacionar contaminantes que têm probabilidade de serem carcinogênicos para a saúde humana ou apresentarem, em estudos com animais, probabilidades carcinogênicas; e/ou Risco Cancerígeno (CR) entre $10^{-4}$ e $10^{-6}$ inclusive.

A categoria cinco (5) de risco corresponde ao enquadramento do contaminante no grupo 1 ou 2A pela IARC (2015); ou A ou B pela USEPA (2015), considerada de efeito gravíssimo ou letal, por relacionar contaminantes que têm comprovada capacidade carcinogênica ou cancerígena para a saúde humana; e/ou Risco Cancerígeno maior que $10^{-4}$; ou para contaminantes não cancerígenos quando $\mathrm{HI}$ for maior que 1 e $\mathrm{ADD}^{*}$ maior ou igual a $\mathrm{DL}_{50}$ (Dose Letal) ou CL50 (Concentração Letal).

\section{Caracterização Qualitativa do Risco para Múltiplos Contaminantes}

A caracterização qualitativa do risco foi realizada para cada elemento da matriz espaçotemporal dos dados de exposição medidos no cenário de contaminação. Os dados da concentração nos compartimentos ambientais, e a respectiva dose de ingresso, foram organizados por ponto espacial de coleta na ordem crescente da distância do ponto central escolhido.

Esse ponto central foi o suposto geradoremissor de poluentes, por exemplo, a pilha de estéril de uma mineradora. Por sua vez, para cada 
ponto espacial foram considerados os dados da evolução temporal da concentração e da dose de ingresso diária. Partindo dessa matriz, foi possível realizar análises temporais e espaciais dos níveis e categorias de risco para o cenário contaminado sob estudo.

Para treinamento da classificação categórica, foi utilizada a técnica de Máquina de Vetores Suporte (SVM), de Vapnik (1992), com o algoritmo kSVM, que constitui uma avançada técnica de aprendizagem supervisionada de máquina que, pela primeira vez, é utilizada na solução desse tipo de problema.

Neste trabalho, foram usados dois conjuntos de dados. Os dados do primeiro ano da avaliação de risco com os indicadores HI, Risco
Cancerígeno (CR) e Classe de Risco Categórica, por contaminante e espaço-temporal, para treinar o ambiente de aprendizagem. E os dados das avaliações de risco dos dois anos seguintes, com os mesmos parâmetros, para o processo de classificação.

O Risco foi categorizado com base nos valores de HI e CR em cada ponto amostral e tempo avaliado. A técnica de SVM classifica binariamente através de um hiperplano geométrico com separação máxima entre as classes, por meio da função decisão na equação 18 e figura 2.

$$
f(\mathrm{x})=\mathrm{w}^{\mathrm{T}} \cdot \mathrm{x}+\mathrm{b}=0
$$

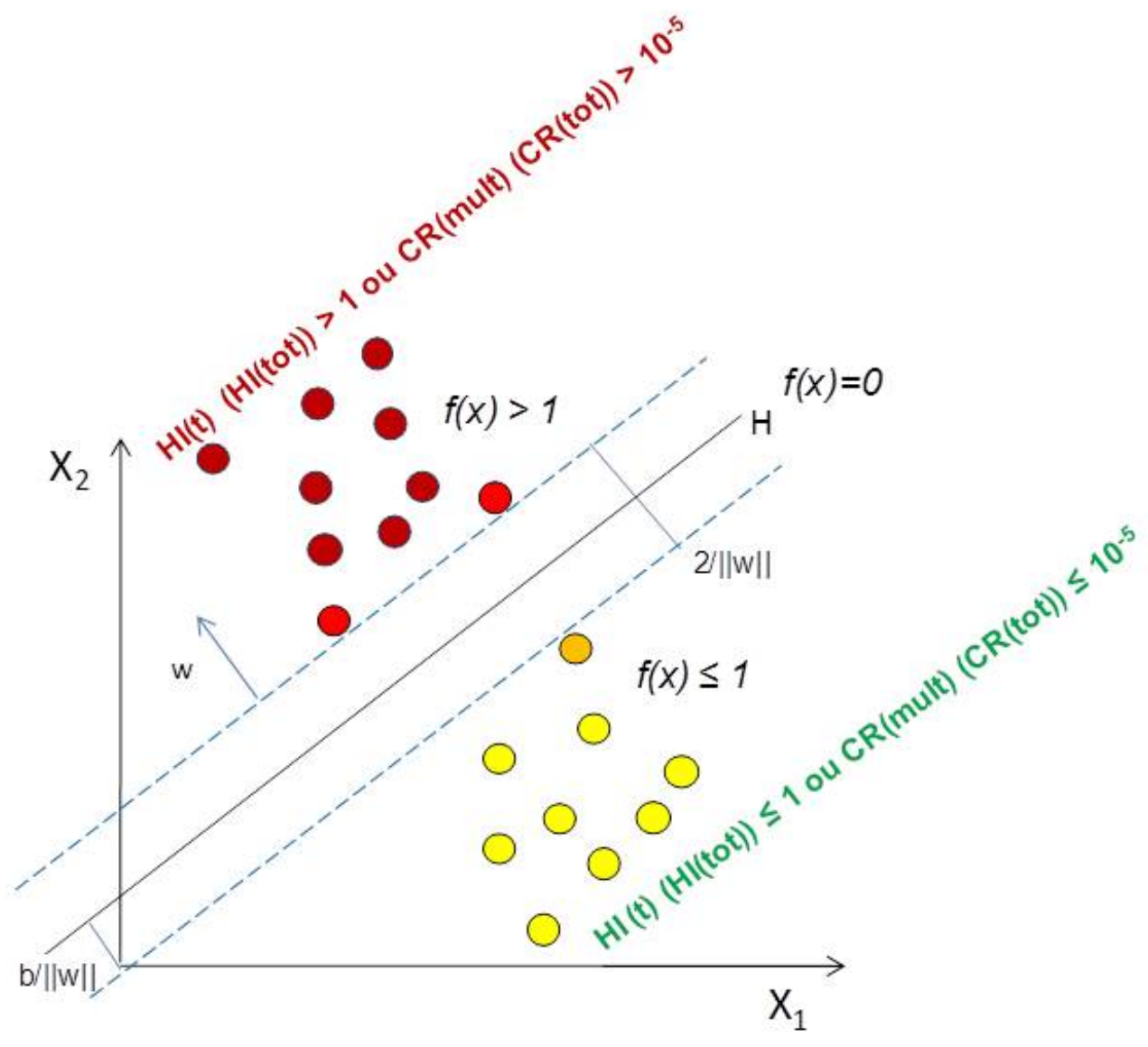

Figura 2 - Hiperplano geométrico de um SVM.

Onde: $\mathrm{x}$ é o vetor de atributos originados de $\mathrm{HI}, \mathrm{CR}$, no espaço e período da amostra, que representam os parâmetros de determinado contaminante, e HItot, CRtot, idem para múltiplos contaminantes em múltiplas rotas. $\mathrm{O}$ w é o vetor de pesos ajustáveis; b é o termo bias. O b/\|w\| é o deslocamento do hiperplano a partir da origem; e $2 /\|w\|$ é a distância entre os hiperplanos separadores das classes.

$\mathrm{O}$ eixo x1 do gráfico, como projeção do vetor $\mathrm{x}$, representa os contaminantes para $\mathrm{o}$ risco individual e o local da amostra para o risco múltiplo; e o eixo x2 representa as classes de $\mathrm{x}$ separadas pelo hiperplano geométrico.

$\mathrm{O}$ exemplo na figura 2 mostra uma classificação binária que ocorre quando $\mathrm{f}(\mathrm{x}) \geq 1$ representa determinada classe para HI (HItot) $>$ 1 (USEPA, 1989) ou CR (CRtot) > 10-5 (CETESB, 2011), e quando $\mathrm{f}(\mathrm{x}) \leq 1$ representa outra classe para HI (HItot) $\leq 1$ ou CR (CRtot) 
$\leq$ 10-5. Algoritmos, como o kSVM, permitem expandir a classificação binária básica para classificação em múltiplas categorias.

Todos os parâmetros dos contaminantes podem ser ajustados em pesos ponderáveis, conforme as características de toxicidade dos contaminantes originadas da EUR.

Quando a substância apresenta parâmetro (HI e CR) mais significativo em razão da correspondência à magnitude da toxicidade, como exemplo de carcinogênico ou cancerígeno, são atribuídos pesos mais elevados (4 ou 5) aplicados na fase de treinamento supervisionado.

Os algoritmos escolhidos para aplicação da multiclassificação foram o SMO (Sequential Minimal Optimization), de Platt (1998), e o MultiClassClassifierUpdateable, de Hall et al. (2009).

A escolha desses algoritmos justifica-se por serem técnicas adequadas para a classificação de dados com poucas categorias ou classes, e que aplicam o método decomposicional o qual resolve subproblemas binários para combinação multiclasse, conforme Faceli et al. (2011).

Considerando a abordagem decomposicional segundo Weston \& Watkins (1998) e Hsu \& Lin (2002) e Xu (2011), é utilizado o método "um-contra-todos", indicado para um conjunto de amostras de pequeno ou médio porte e adequado para dados de avaliação de risco com limitada quantidade de contaminantes e rotas de exposição.

Para o teste de significância estatística entre os classificadores kSVM são aplicados o índice Kappa (k), erro absoluto e relativo médio, erro médio quadrático e matriz de confusão. Todos calculados usando o software Weka (Waikato Environment for Knowledge Analysis), versão 3.7.5, especificado por Hall et al. (2009), Wood (2007) e Anderson et al. (2007).

\section{RESULTADOS E DISCUSSÕES}

Na tabela 2 é mostrado o conjunto de valores assumidos das concentrações dos contaminantes, na matriz espaço temporal selecionada para o estudo. Os valores destas concentrações, no primeiro período 2005-2007, foram tomados dos dados reportados por Lima (2009). A simulação da evolução temporal das concentrações dos contaminantes foi realizada de acordo com as equações 5 e 6 . As equações para avaliação da exposição foram simuladas para o cenário residencial e seus moradores como receptores potenciais.

No cálculo foram incluídas as mesmas rotas e equações de transferência e exposição consideradas no trabalho de Lima (2009). Assim, foram incluídas as seguintes rotas de exposição: inalação de material particulado, contato dérmico com solo, ingestão acidental de solo, ingestão de água subterrânea, contato dérmico com água, ingestão de hortaliças (alface, couve, abóbora) e ingestão de vegetais (frutos, aveia, trigo, milho, feijão, batatas), de possível cultivo na região nos cenários estudados, para as vias de ingresso por ingestão oral, inalação e contato dérmico.

Para a ingestão das hortaliças e vegetais foram utilizados Fatores de Biotransferência (BTF) segundo Golder (2005), USEPA (1997) e Baes et al. (1984).
Para realizar adequadamente a comparação do método de avaliação de risco aplicado por Lima (2009) com o método SysRisk proposto no presente trabalho, em ambos foram acompanhadas as mesmas suposições para estimar a exposição e em particular as taxas de ingestão de hortaliças e vegetais, as quais foram baseadas na média ou características padrão de exposição, definidas pela CETESB (2001), USEPA (1989, 1997 e 2004) e HEALTH CANADA (2012). Os resultados dos cálculos de riscos por múltiplas rotas para cada metal, e do risco total, são mostrados nas figuras 3 e 4 . Os cálculos mostrados nas figuras 3a, 3b, 4a e 4c foram realizados com base no APD (equação 3) para focalizar a contribuição parcial de cada subperíodo do tempo ao total do risco.

A evolução decrescente no cálculo por SysRisk, das grandezas de riscos ilustradas nas barras das figuras 3 e 4, responde à redução na concentração simulada para acompanhar o cenário de descomissionamento com diminuição das atividades operacionais da metalúrgica.

A figura 3a mostra a classificação de Risco Categórico de acordo com a EUR, em valores máximos para HI(t) com evolução temporal, por substância e rotas simultâneas de exposição. A figura 3b, da mesma forma, ilustra a classificação do $\mathrm{CR}^{\text {mult }}$ pela EUR, com a 
evolução temporal nos períodos avaliados.

$\mathrm{Na}$ figura $3 \mathrm{a}$, observa-se que o risco da exposição a metais não cancerígenos (Fe e Zn), no gráfico (a), foi classificado na categoria de risco 1 - nenhum efeito -, em razão da apuração do HI abaixo de 1,0. Segundo a WHO-IPCS (2015), a dose oral fatal de sulfato de zinco tem sido estimada em
10 - 30g por kg de peso corpóreo, o que o torna um contaminante de toxicidade crônica pela longa exposição, caso possível em uma metalurgia de beneficiamento própria de zinco.

Comparando-se a caracterização do risco individual, o Zn obteve valores maiores do que o Fe, mesmo não participando de todas as rotas de exposição.

Tabela 2 - Concentrações dos contaminantes estimados para três anos.

\begin{tabular}{|c|c|c|c|c|c|c|c|c|c|}
\hline \multirow[t]{2}{*}{ Período } & \multirow[t]{2}{*}{ Local } & \multicolumn{4}{|c|}{ Solo $\left(\mathrm{mg} \mathrm{kg}^{-1}\right)$} & \multicolumn{4}{|c|}{ Áqua $\left(\mathrm{mg} \mathrm{L}^{-1}\right)$} \\
\hline & & $\mathrm{Fe}$ & $\mathrm{Zn}$ & Co & $\mathrm{Pb}$ & $\mathrm{Fe}$ & $\mathrm{Zn}$ & Co & $\mathrm{Pb}$ \\
\hline \multirow{3}{*}{$2005-2007$} & A1 & $3,80 \mathrm{E}+03$ & $6,73 E+05$ & $\mathrm{n}$ & $3,80 \mathrm{E}+03$ & $6,05 E+01$ & $7,66 \mathrm{E}+01$ & $3,00 \mathrm{E}-02$ & $5,80 \mathrm{E}-01$ \\
\hline & A2 & $3,70 \mathrm{E}+03$ & $1,29 \mathrm{E}+05$ & $n$ & $1,20 \mathrm{E}+03$ & $1,87 \mathrm{E}+01$ & $6,46 \mathrm{E}+00$ & $4,00 \mathrm{E}-03$ & $2,00 \mathrm{E}-03$ \\
\hline & A3 & $3,40 \mathrm{E}+03$ & $3,10 \mathrm{E}+04$ & $\mathrm{n}$ & $3,06 \mathrm{E}+02$ & $3,27 \mathrm{E}+01$ & $5,89 \mathrm{E}+00$ & 4,00E-03 & 2,00E-03 \\
\hline \multirow{3}{*}{ 2007-2009 } & A1 & $3,10 \mathrm{E}+03$ & $5,38 \mathrm{E}+05$ & $\mathrm{n}$ & $3,08 E+03$ & $4,85 E+01$ & $6,22 \mathrm{E}+01$ & $2,50 \mathrm{E}-02$ & $4,66 \mathrm{E}-01$ \\
\hline & $\mathrm{A} 2$ & $3,02 E+03$ & $1,03 \mathrm{E}+05$ & $n$ & $9,96 \mathrm{E}+02$ & $1,50 \mathrm{E}+01$ & $6,17 \mathrm{E}+00$ & $\mathrm{n}$ & $\mathrm{n}$ \\
\hline & A3 & $2,78 \mathrm{E}+03$ & $2,49 \mathrm{E}+04$ & $n$ & $2,81 E+02$ & $2,62 \mathrm{E}+01$ & $\mathrm{n}$ & $\mathrm{n}$ & $\mathrm{n}$ \\
\hline \multirow{3}{*}{ 2009-2011 } & A1 & $2,40 \mathrm{E}+03$ & $4,04 \mathrm{E}+05$ & $\mathrm{n}$ & $2,35 \mathrm{E}+03$ & $3,64 \mathrm{E}+01$ & $4,79 \mathrm{E}+01$ & $2,00 \mathrm{E}-02$ & $3,52 \mathrm{E}-01$ \\
\hline & A2 & $2,34 \mathrm{E}+03$ & $7,76 \mathrm{E}+04$ & $\mathrm{n}$ & $7,92 \mathrm{E}+02$ & $1,13 \mathrm{E}+01$ & $5,88 \mathrm{E}+00$ & $\mathrm{n}$ & $\mathrm{n}$ \\
\hline & A3 & $2,16 \mathrm{E}+03$ & $1,88 \mathrm{E}+04$ & $n$ & $2,56 \mathrm{E}+02$ & $1,97 \mathrm{E}+01$ & $\mathrm{n}$ & $\mathrm{n}$ & $\mathrm{n}$ \\
\hline \multirow{3}{*}{ 2011-2013 } & A1 & $1,70 \mathrm{E}+03$ & $2,69 \mathrm{E}+05$ & $\mathrm{n}$ & $1,63 \mathrm{E}+03$ & $2,44 \mathrm{E}+01$ & $3,36 \mathrm{E}+01$ & $1,50 \mathrm{E}-02$ & $2,38 \mathrm{E}-01$ \\
\hline & $\mathrm{A} 2$ & $1,66 \mathrm{E}+03$ & $5,19 \mathrm{E}+04$ & $n$ & $5,88 \mathrm{E}+02$ & $7,64 \mathrm{E}+00$ & $5,58 \mathrm{E}+00$ & $\mathrm{n}$ & $\mathrm{n}$ \\
\hline & A3 & $1,54 \mathrm{E}+03$ & $1,27 \mathrm{E}+04$ & $\mathrm{n}$ & $2,30 \mathrm{E}+02$ & $1,32 \mathrm{E}+01$ & $\mathrm{n}$ & $\mathrm{n}$ & $\mathrm{n}$ \\
\hline \multirow{3}{*}{ 2013-2015 } & A1 & $1,00 \mathrm{E}+03$ & $1,35 \mathrm{E}+05$ & $n$ & $9,04 \mathrm{E}+02$ & $1,23 \mathrm{E}+01$ & $1,93 \mathrm{E}+01$ & $1,00 \mathrm{E}-02$ & $1,24 \mathrm{E}-01$ \\
\hline & A2 & $9,80 \mathrm{E}+02$ & $2,62 \mathrm{E}+04$ & $\mathrm{n}$ & $3,84 \mathrm{E}+02$ & $3,97 \mathrm{E}+00$ & $\overline{5,29 \mathrm{E}+00}$ & $\mathrm{n}$ & $\mathrm{n}$ \\
\hline & A3 & $9,20 \mathrm{E}+02$ & $6,56 \mathrm{E}+03$ & $\mathrm{n}$ & $2,05 \mathrm{E}+02$ & $6,77 \mathrm{E}+00$ & $\mathrm{n}$ & $\mathrm{n}$ & $n$ \\
\hline \multicolumn{2}{|c|}{ Referência (CETESB 2005) } & $3,00 \mathrm{E}+02$ & $4,50 \mathrm{E}+02$ & $3,50 \mathrm{E}+01$ & $1,80 \mathrm{E}+02$ & $3,00 \mathrm{E}-01$ & $5,00 \mathrm{E}+00$ & 5,00E-03 & $1,00 \mathrm{E}-02$ \\
\hline \multirow[t]{2}{*}{ Período } & \multirow[t]{2}{*}{ Local } & \multicolumn{4}{|c|}{ Hortaliças (mg kg ${ }^{-1}$ ) } & \multicolumn{4}{|c|}{ Vegetais (mg kg $\left.{ }^{-1}\right)$} \\
\hline & & $\mathbf{F e}$ & $\mathrm{Zn}$ & Co & $\mathbf{P b}$ & $\mathbf{F e}$ & Zn & Co & $\mathbf{P b}$ \\
\hline \multirow{3}{*}{ 2005-2007 } & A1 & $3,19 \mathrm{E}+00$ & $1,52 \mathrm{E}+05$ & $4,10 \mathrm{E}-03$ & $2,92 \mathrm{E}+01$ & 7,97E-01 & $9,15 \mathrm{E}+04$ & $1,40 \mathrm{E}-03$ & $5,83 \mathrm{E}+00$ \\
\hline & $\overline{\mathrm{A} 2}$ & $2,50 \mathrm{E}+00$ & $2,91 \mathrm{E}+04$ & $5,00 \mathrm{E}-04$ & $8,11 \mathrm{E}+00$ & $6,25 \mathrm{E}-01$ & $1,75 \mathrm{E}+04$ & $2,00 \mathrm{E}-04$ & $1,62 \mathrm{E}+00$ \\
\hline & A3 & $2,53 E+00$ & $7,06 \mathrm{E}+03$ & 5,00E-04 & $2,08 \mathrm{E}+00$ & 6,32E-01 & $4,23 E+03$ & $2,00 \mathrm{E}-04$ & $4,16 \mathrm{E}-01$ \\
\hline \multirow{3}{*}{ 2007-2009 } & A1 & $2,58 \mathrm{E}+00$ & $1,23 \mathrm{E}+05$ & $3,28 \mathrm{E}-03$ & $2,37 \mathrm{E}+01$ & $6,46 \mathrm{E}-01$ & $7,41 \mathrm{E}+04$ & $1,15 \mathrm{E}-03$ & $4,72 E+00$ \\
\hline & $\mathrm{A} 2$ & $2,03 E+00$ & $2,36 \mathrm{E}+04$ & 4,37E-04 & $6,57 \mathrm{E}+00$ & $5,06 \mathrm{E}-01$ & $1,42 \mathrm{E}+04$ & $1,53 \mathrm{E}-04$ & $1,31 \mathrm{E}+00$ \\
\hline & $\overline{\mathrm{A} 3}$ & $2,05 \mathrm{E}+00$ & $5,72 \mathrm{E}+03$ & $4,40 \mathrm{E}-04$ & $1,68 \mathrm{E}+00$ & $5,12 \mathrm{E}-01$ & $3,43 \mathrm{E}+03$ & $1,50 \mathrm{E}-04$ & $3,37 \mathrm{E}-01$ \\
\hline \multirow{3}{*}{ 2009-2011 } & $\mathrm{A} 1$ & $2,09 \mathrm{E}+00$ & $9,97 \mathrm{E}+04$ & $2,66 \mathrm{E}-03$ & $1,92 \mathrm{E}+01$ & $5,23 \mathrm{E}-01$ & $6,00 \mathrm{E}+04$ & 9,32E-04 & $3,83 E+00$ \\
\hline & $\mathrm{A} 2$ & $1,64 \mathrm{E}+00$ & $1,91 \mathrm{E}+04$ & $3,50 \mathrm{E}-04$ & $5,32 \mathrm{E}+00$ & $4,10 \mathrm{E}-01$ & $1,15 E+04$ & $1,20 \mathrm{E}-04$ & $1,06 \mathrm{E}+00$ \\
\hline & $\overline{\mathrm{A} 3}$ & $1,66 \mathrm{E}+00$ & $4,63 \mathrm{E}+03$ & $3,50 \mathrm{E}-04$ & $1,36 \mathrm{E}+00$ & $4,15 \mathrm{E}-01$ & $2,78 \mathrm{E}+03$ & $1,20 \mathrm{E}-04$ & 2,73E-01 \\
\hline \multirow{3}{*}{ 2011-2013 } & A1 & $1,70 \mathrm{E}+00$ & $8,08 \mathrm{E}+04$ & $2,15 \mathrm{E}-03$ & $1,55 \mathrm{E}+01$ & $4,24 \mathrm{E}-01$ & $4,86 \mathrm{E}+04$ & $7,50 \mathrm{E}-04$ & $3,10 \mathrm{E}+00$ \\
\hline & $\mathrm{A} 2$ & $1,33 \mathrm{E}+00$ & $1,55 \mathrm{E}+04$ & $2,90 \mathrm{E}-04$ & $4,31 \mathrm{E}+00$ & $3,32 \mathrm{E}-01$ & $9,30 \mathrm{E}+03$ & $1,00 \mathrm{E}-04$ & $8,61 \mathrm{E}-01$ \\
\hline & A3 & $1,34 \mathrm{E}+00$ & $3,75 \mathrm{E}+03$ & $2,90 \mathrm{E}-04$ & $1,11 \mathrm{E}+00$ & $3,36 \mathrm{E}-01$ & $2,25 \mathrm{E}+03$ & $1,00 \mathrm{E}-04$ & $2,21 \mathrm{E}-01$ \\
\hline \multirow{3}{*}{ 2013-2015 } & $\mathrm{A} 1$ & $1,37 \mathrm{E}+00$ & $6,54 \mathrm{E}+04$ & $1,74 \mathrm{E}-03$ & $1,26 \mathrm{E}+01$ & $3,43 \mathrm{E}-01$ & $3,94 \mathrm{E}+04$ & $6,10 \mathrm{E}-04$ & $2,51 \mathrm{E}+00$ \\
\hline & $\mathrm{A} 2$ & $1,08 \mathrm{E}+00$ & $1,25 \mathrm{E}+04$ & $2,30 \mathrm{E}-04$ & $3,49 \mathrm{E}+00$ & $2,69 \mathrm{E}-01$ & $7,53 \mathrm{E}+03$ & $8,10 \mathrm{E}-05$ & $6,97 \mathrm{E}-01$ \\
\hline & A3 & $1,09 \mathrm{E}+00$ & $3,04 \mathrm{E}+03$ & $2,00 \mathrm{E}-04$ & 8,95E-01 & 2,72E-01 & $1,82 \mathrm{E}+03$ & $8,00 \mathrm{E}-05$ & $1,79 \mathrm{E}-01$ \\
\hline
\end{tabular}

n: não disponível.

Essa situação era esperada por tratar-se de uma exploradora de minério de zinco e pela associação entre o ferro e o zinco, tanto na composição natural quanto no processo de beneficiamento. $\mathrm{O}$ ferro não é classificado como carcinogênico. Em WHO-IPCS (2015) é relatado algum efeito adverso no funcionamento metabólico e acúmulo de traços no fígado de humanos, para exposição 
crônica com dose acima de $0,8 \mathrm{mg} \mathrm{kg} \mathrm{dia}^{-1}$, embora, para outras fontes, não haja relato de estudos toxicológicos com ferro.

E há o aumento no HI do último período, iniciado em 2015, em razão do efeito cumulativo da dose de ingresso para a duração maior da exposição de 14 anos, complementar ao período referencial (24 anos) para residentes

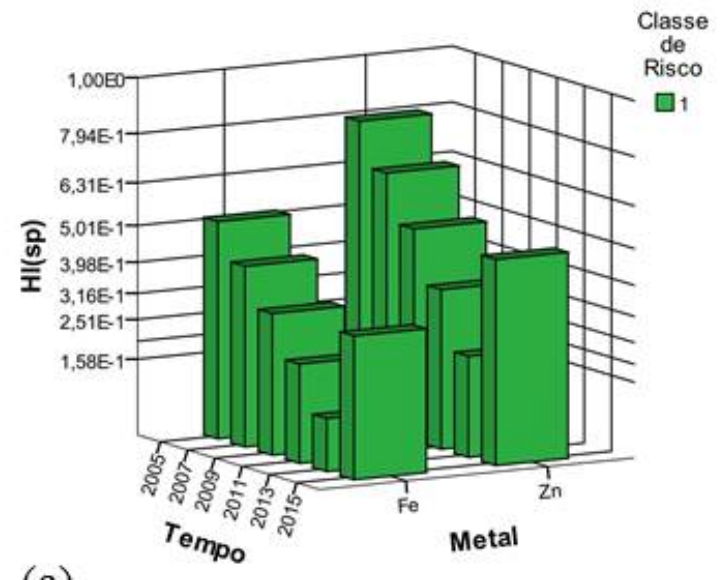

(a)

Avaliação para metais não cancerígenos por exposição acumulada no tempo

a $\mathrm{Fe} \mathrm{Zn}$

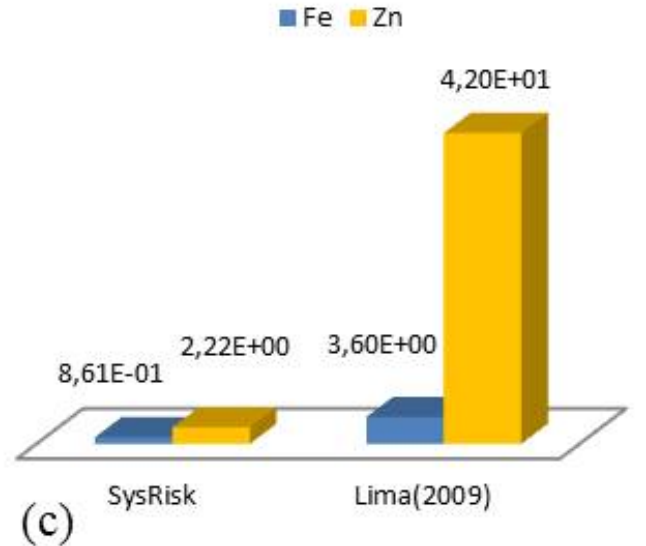

do local afetado.

A figura 3b ilustra os metais que apresentam $\mathrm{CR}^{\text {mult }}$ considerando as múltiplas rotas de exposição. O cobalto é registrado como possível carcinogênico no grupo 2B pelo IARC (2015). Comparando-se os níveis do Risco Cancerígeno $\left(\mathrm{CR}^{\mathrm{mult}}\right), \quad$ o $\mathrm{Co}$ apresentou moderada indicação.

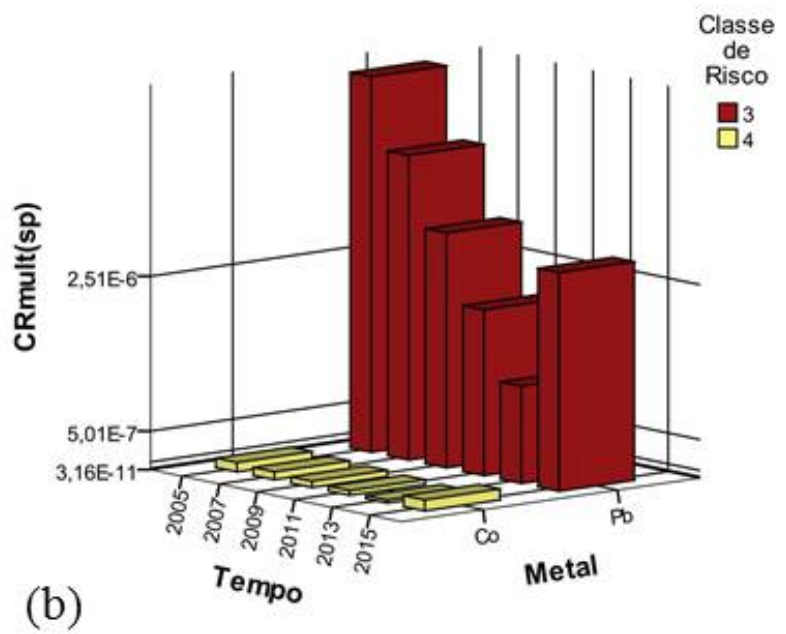

Avaliação para metais cancerígenos por exposição acumulada no tempo

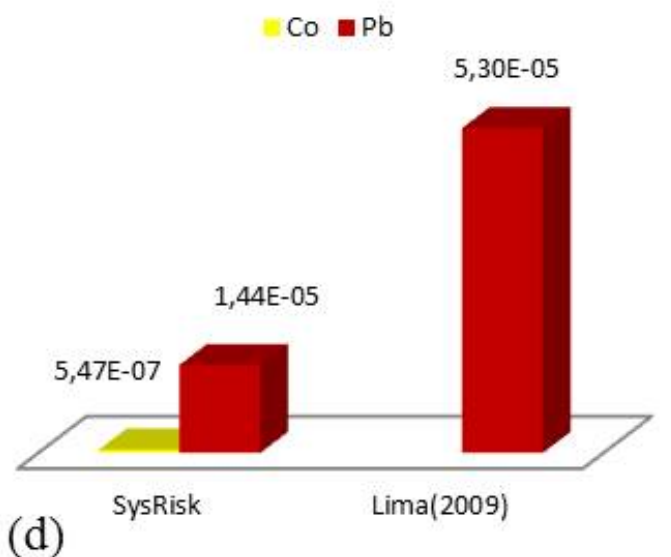

Figura 3 - Comparativo de Risco Toxicológico por Contaminante: a) comparativo entre gráficos do Risco Categórico

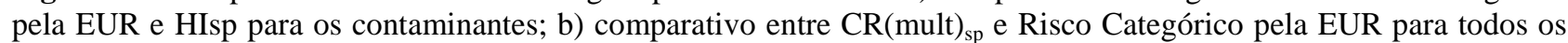
contaminantes; c) avaliação para não cancerígenos acumulada por rota de exposição; d) avaliação para cancerígenos acumulada por rotas de exposição.

de risco com probabilidade próxima a $10^{-6}$ de desenvolver câncer para a população exposta. É classificado por peso de evidência no grupo 2B do IARC (2015) como possível carcinogênico.

Por esta razão, embora o Co tenha apresentado HI com valor menor que 1,0, foi classificado pela EUR na categoria 3 nos períodos e ponto amostrais (2005 - A2 e A3, 2009 - A1, 2011 - A1, 2013 - A1 e 2015 - A1), e na categoria 4 nos períodos e pontos amostrais (2005 - A1, 2007 - A1, A2 e A3, 2009, 2011, 2013 e 2015 - A2 e A3).

O chumbo é classificado pela USEPA-IRIS (2015) como carcinogênico, no grupo B2 provável cancerígeno para seres humanos. É considerado de toxicidade crônica, que provoca vários efeitos adversos à saúde, muitos destes irreversíveis e mutagênicos. 
Pode-se observar, na figura 3b, que a exposição de $\mathrm{Pb}$ apresenta elevado risco inclusive em baixa concentração de exposição na água de beber ou no ar, com risco de câncer de 1 em 10.000 casos de exposição.

Sua caracterização apresentou risco considerável de aproximadamente $10^{-6}$ de $\mathrm{CR}^{\text {mult }}$, com elevada probabilidade de desenvolver câncer nos períodos e pontos amostrais (2005, 2007, 2009, 2011, 2013, 2015 - A1). Apresentou HI inferior a 1,0 em todos os períodos e pontos amostrais e alcançou a classe 3 e 4 da EUR para os períodos e pontos amostrais, indicando elevada probabilidade de gerar efeitos adversos crônicos diante da exposição frequente.

Os resultados ilustrados na figura 3 mostram também a classificação do Risco Categórico

Índice de perigo total $\mathrm{HI}_{\mathrm{sp}}$ por espaço-temporal

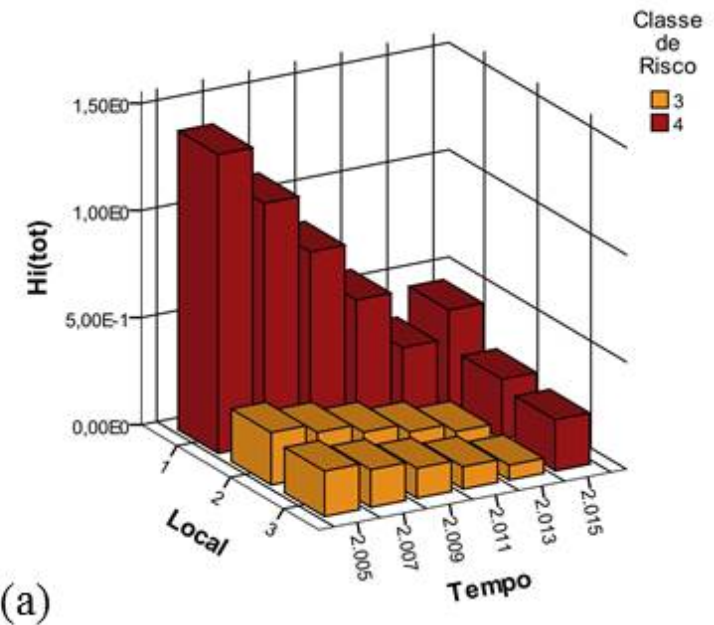

Índice de perigo total $-\mathrm{HI}$ (tot)

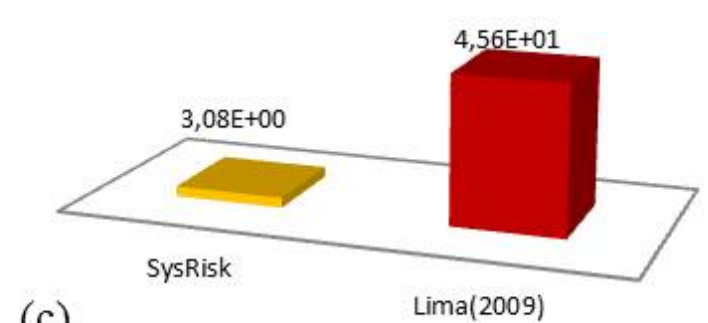

(c)

$\operatorname{Lima}(2009)$ obtido por SysRisk para todos os contaminantes, numa caracterização qualitativa que pode oferecer informações adicionais além dos índices quantitativos HI e Risco Cancerígeno. A classificação determinada pode indicar a possibilidade de ocorrência de efeitos de vários níveis adversos à saúde e morbidade pela exposição às substâncias nocivas.

A caracterização do risco considerou, na aproximação aditiva, a associação simultânea pela sinergia dos efeitos químicos e toxicológicos causados pela múltipla exposição dos contaminantes, nos espaços amostrais e nos períodos avaliados. A figura 4 mostra essa caracterização do risco pela abordagem quantitativa dos indicadores $\mathrm{HI}^{\text {tot }}$ e $\mathrm{CR}^{\text {tot }}$, e pela abordagem qualitativa do risco categórico ordinal classificado por kSVM.

\section{Risco cancerígeno total $\mathrm{CR}$ (tot) $)_{\mathrm{sp}}$ por espaço- temporal}

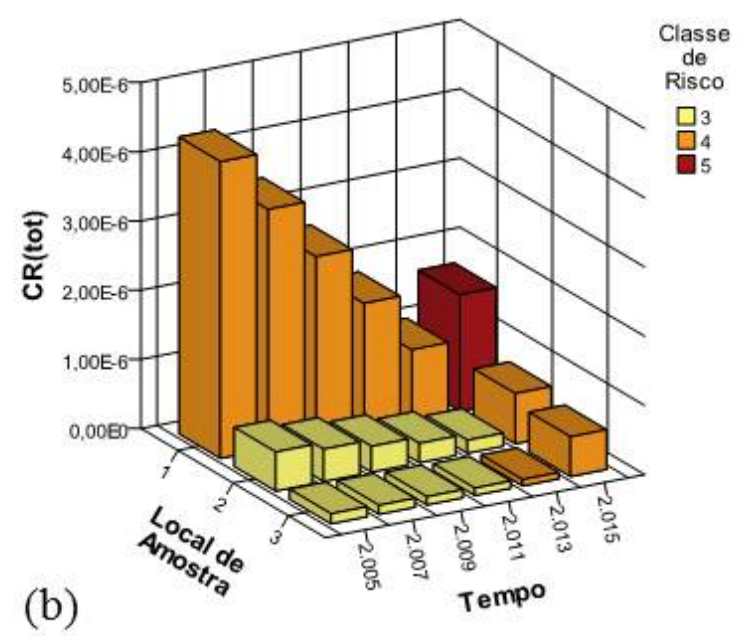

Risco cancerígeno total - CR(tot)

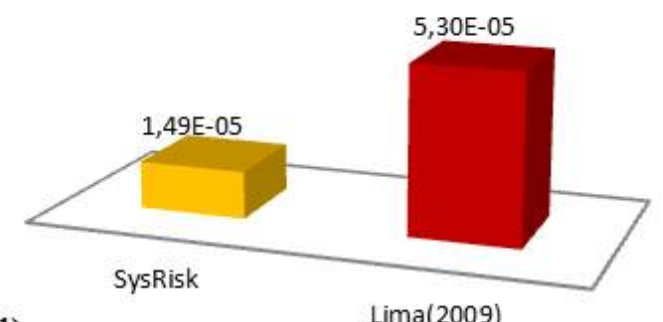

(d)

Figura 4 - a) Contribuição por subperíodo ao índice de perigo múltiplo na matriz espaço-temporal; b) Contribuição por subperíodo ao Risco Cancerígeno Total na matriz elemento espaço-temporal; c) Comparação da avaliação de risco por exposição acumulada no período integral de exposição para não cancerígenos; d) Comparação da avaliação de risco por exposição acumulada no período integral de exposição para cancerígenos. 
$\mathrm{Na}$ figura 4a observa-se que o maior risco, da classe 4, aparece no local A1 em todos os períodos avaliados e para todos os pontos amostrais no período residual de 14 anos, iniciado em 2015.

Os riscos foram categorizados pela EUR nas classes 3 e 4 em razão da presença simultânea dos contaminantes cancerígenos com metais não carcinogênicos. Porém, embora tenha ocorrido risco elevado, somente no ponto A1, para os períodos 2005 a 2009, e para o período residual iniciado em 2015, ocorreram HI superiores a 1,0 em vários locais e tempos de amostra.

$\mathrm{Na}$ figura $4 \mathrm{~b}$ foi evidenciado perigo cancerígeno para probabilidades de risco superiores a $10^{-6}$, com classificação nas categorias 3, 4 e 5 .

No período de 14 anos, iniciado em 2015, ocorreu risco categórico na classe 5, devido ao somatório dos efeitos simultâneos para 0 período acumulativo da exposição.

Em geral, os resultados ilustrados na figura 4 mostram que quando é avaliada a contribuição simultânea de várias rotas de exposição e diferentes substâncias, os riscos aumentam consideravelmente, constatando que, praticamente em todos os elementos da matriz espaçotemporal estudada, são obtidas as categorias mais altas 3, 4 e 5 para o índice de risco.

Nas figuras 3c e 3d, é mostrada a comparação do risco cumulativo para o período integral de 24 anos de duração da exposição para os receptores residentes. A figura 3c ilustra o comportamento da caracterização de risco por HI para Fe e Zn, calculado pelo SysRisk e no trabalho de Lima (2009).

O gráfico (d) ilustra a caracterização por Risco Cancerígeno múltiplo para $\mathrm{Co}$ e $\mathrm{Pb}$, ressaltando que, no trabalho de Lima (2009), o Co não foi considerado como possível cancerígeno.

Observa-se, em ambas as figuras, um comportamento similar, com valores elevados de risco nos resultados de Lima (2009). Nos resultados do SysRisk há indicação de risco na caracterização quantitativa, porém com valores menores.

Os resultados obtidos e mostrados nas figuras 3 e 4 foram baseados em um subconjunto do estudo completo da contaminação ambiental da metalúrgica de $\mathrm{Zn}$, realizado por Lima (2009). Por isso este trabalho tem apenas um caráter relativo, mas que permite mostrar o potencial de resultados que a nova proposta SysRisk é capaz de produzir.

Por exemplo, para o cenário avaliado, na abordagem SysRisk, é possível notar que o local A1 deverá ser motivo das maiores atenções no plano de gerenciamento de riscos. Por outro lado, a sequência decrescente dos riscos, em todo o espaço contaminado, pode permitir organizar, em cada ano, as ações de mitigação de forma mais eficiente e, assim, atender as necessidades reais do cenário atingido.

\section{Treinamento e Classificação da Avaliação de Risco}

Os dados da exposição acumulada para o período integral da avaliação de risco foram aplicados para treinar a kSVM. A seguir, foram classificados, para todos os períodos, o risco por contaminante individual e o risco múltiplo na matriz espaço-temporal.

Foram aplicados os algoritmos MultiClass ClassifierUpdateable, de Hall et al. (2009) e SMO, de Platt (1998), utilizando-se o método de classificação decomposicional. Os resultados estatísticos estão ilustrados na tabela 3. Para treinamento do kSVM foi utilizado o conjunto do período integral de 24 anos para duração da exposição, considerando todos os períodos e locais de amostras, e para testar a classificação foram utilizados os dados dos períodos iniciados em 2007 e 2013.

Observa-se que o melhor conjunto de treinamento foi alcançado pelo método $S M O$, com treinamento correto em $94,4 \%$ das instâncias dos dados avaliativos de risco. O mesmo método obteve melhores classificações para os dois períodos testados com 91,7\% (Kappa de 83\%) de instâncias classificadas corretamente.

Como se observa na tabela 3 , para os dois métodos empregados, os dados do segundo período (2013) obtiveram resultados idênticos, com 11 instâncias classificadas corretamente e índice Kappa de 0,86.

Da mesma forma, a classificação do período 2007 para SMO obteve o mesmo desempenho, porém, com melhor indicador na média de precisão da amostra, com valor de 0,94. Todos os resultados alcançados são considerados significativos.

O indicador da Curva ROC (Hanley \& McNeil, 1982) aponta precisão das amostras 
superior a $92 \%$ para todas as classes treinadas, e acima de 0,87 para a média de precisão nos dados preditos na classificação. Para o erro absoluto médio foi obtido o mínimo de 0,23; e para o erro quadrático médio, o mínimo de 0,31 (pelo SMO) durante as classificações dos dados nos períodos de teste, significando que, embora tenham realizado classificações corretas, o nível de acertos na classificação não necessariamente permaneceria no mesmo patamar. Pela matriz de confusão, para a fase de treinamento, podese verificar que 36 ocorrências na $1^{\text {a }}$ linha foram classificadas corretamente na classe de Risco Categórico 1, para os dois métodos.

No conjunto, não houve ocorrência de classe de risco 2, havendo erro de predição pelo método MultiClassClassifierUpdateable nesta categoria. Para as classes de riscos 3 e 4, as classificações pelos dois métodos apresentaram erros de predição, sendo 2 e 3 no primeiro método, e 3 e 1 no segundo, respectivamente para cada técnica aplicada. Nesse caso, pode haver necessidade de ajustamento do modelo ou pesos da máquina de aprendizagem, ou manutenção na classificação pela EUR. Para a classe de risco 5, não houve nenhuma ocorrência no conjunto apresentado.

O classificador SMO apresentou melhores resultados, indicando ser a melhor técnica para tratar os dados avaliados, pois obteve melhor desempenho durante o treinamento e na classificação, alcançando resultados uniformes para os dois períodos avaliados.

Como pode ser observado na tabela 3 , a técnica SMO mostrou um ajuste estatístico melhor.

Tabela 3 - Resultado estatístico do treinamento e classificação de risco.

\begin{tabular}{|c|c|c|c|c|c|c|c|c|c|c|c|c|c|c|}
\hline \multirow{2}{*}{\multicolumn{3}{|c|}{$\begin{array}{l}\text { Algoritmos: } \\
\text { Indicadores }\end{array}$}} & \multicolumn{6}{|c|}{ MultiClassClassifierUpdateable } & \multicolumn{6}{|c|}{ SMO } \\
\hline & & & $\begin{array}{l}\text { Treino } \\
\text { (2015) }\end{array}$ & & $\begin{array}{l}\text { este } \\
007)\end{array}$ & & & & $\begin{array}{l}\text { Treino } \\
\text { (2015) }\end{array}$ & & $\begin{array}{l}\text { Teste } \\
(2007)\end{array}$ & & & \\
\hline \multicolumn{3}{|c|}{ Instâncias corretamente classificadas } & $\begin{array}{c}67 \\
(93,1 \%)\end{array}$ & & 10 & \multicolumn{3}{|c|}{$\begin{array}{c}11 \\
(91,7 \%)\end{array}$} & $\begin{array}{c}68 \\
(94,4 \%)\end{array}$ & \multicolumn{3}{|c|}{$\begin{array}{c}11 \\
(91,7 \%)\end{array}$} & \multicolumn{2}{|c|}{$\begin{array}{c}11 \\
(91,7 \%)\end{array}$} \\
\hline \multicolumn{3}{|c|}{ Instâncias incorretamente classificadas } & $5(6,9 \%)$ & $2(1$ & $6,7 \%)$ & \multicolumn{3}{|c|}{$1(8,3 \%)$} & $4(5,6 \%)$ & \multicolumn{3}{|c|}{$1(8,3 \%)$} & \multicolumn{2}{|c|}{$1(8,3 \%)$} \\
\hline \multicolumn{3}{|c|}{ Kappa estatístico } & 0,89 & & 73 & & \multicolumn{2}{|c|}{0,86} & 0,91 & \multicolumn{3}{|c|}{0,86} & \multicolumn{2}{|c|}{0,86} \\
\hline \multicolumn{3}{|c|}{ Erro absoluto médio } & 0,29 & & ,29 & & \multicolumn{2}{|c|}{0,29} & 0.23 & \multicolumn{3}{|c|}{0,24} & \multicolumn{2}{|c|}{0.24} \\
\hline \multicolumn{3}{|c|}{ Erro quadrático médio } & 0,36 & & 36 & & \multicolumn{2}{|c|}{0,36} & 0.31 & \multicolumn{3}{|c|}{0,33} & \multicolumn{2}{|c|}{0.33} \\
\hline \multicolumn{3}{|c|}{ Média da precisão da amostra } & 0,93 & & 92 & & \multicolumn{2}{|c|}{0,87} & 0,94 & \multicolumn{3}{|c|}{0,92} & \multicolumn{2}{|c|}{0,87} \\
\hline \multicolumn{3}{|c|}{ Cobertura de casos (0,95 nível) } & $100 \%$ & & $0 \%$ & & \multicolumn{2}{|c|}{$100 \%$} & $100 \%$ & \multicolumn{3}{|c|}{$100 \%$} & \multicolumn{2}{|c|}{$100 \%$} \\
\hline \multicolumn{3}{|c|}{ Número total de instâncias } & 72 & & 12 & & \multicolumn{2}{|c|}{12} & 72 & & 12 & & & \\
\hline Curva Ro & do treinam & & & & & a & $\mathrm{riz} \mathrm{C}$ & nfus & do treinam & & & & & \\
\hline Classe & MultiClass & SMO & Classes & 1 & 2 & 3 & 4 & 5 & Classes & 1 & 2 & 3 & 4 & 5 \\
\hline 1 & 0,98 & 1,0 & 1 & 36 & 0 & 0 & 0 & 0 & 1 & 36 & 0 & 0 & 0 & 0 \\
\hline 2 & 0,93 & - & 2 & 1 & 0 & 0 & 0 & 0 & 2 & 0 & $\mathbf{0}$ & 0 & 0 & 0 \\
\hline 3 & 1,0 & 0,92 & 3 & 0 & 0 & 9 & 2 & 0 & 3 & 0 & 0 & 8 & 3 & 0 \\
\hline 4 & 1,0 & 0,95 & 4 & 0 & 0 & 3 & 2 & 0 & 4 & 0 & 0 & 1 & 2 & 0 \\
\hline 5 & 1,0 & - & & & & & 2 & & & & & & 4 & \\
\hline & & & 5 & 0 & 0 & 0 & 0 & 0 & 5 & 0 & 0 & 0 & 0 & 0 \\
\hline
\end{tabular}

A avaliação qualitativa dos riscos, obtida neste estudo, qualificou os locais e períodos amostrais dos níveis mais elevados 3,4 e 5 . Esta informação mostra ter uma forma mais adequada para comunicar os riscos à população e tomadores de decisão. O conjunto de parâmetros que constituem a avaliação quantitativa, como HI, CR e grandezas derivadas, que obviamente são importantes para a elaboração técnica dos planos de mitigação, neste caso, são resumidos em categorias de níveis de risco de fácil interpretação.

Além disso, a classificação ordinal dos riscos, obtida para o cenário estudado, mostra que apenas com a consideração parcial de 4 metais, as classificações de riscos cumulativos de todas as substâncias diferem significativamente dos riscos dos elementos químicos individuais. Quando estudadas as substâncias de forma individual, foram obtidos níveis 1 para contaminantes não cancerígenos e níveis 3 e 4 para cancerígenos. 
$\mathrm{Na}$ avaliação dos riscos da ação simultânea de elementos químicos não cancerígenos, os níveis se elevaram a valores 3 e 4 , e para substâncias cancerígenas foram obtidos níveis de 3 , 4 e 5 .
Desta forma, podemos concluir que a abordagem qualitativa dos riscos, proposta no SysRisk, permite mostrar claramente o efeito sinérgico dos riscos cumulativos derivados da exposição simultânea a vários contaminantes.

\section{CONCLUSÃO}

A nova metodologia SysRisk, proposta e aplicada, mostrou ser uma ferramenta que permitiu realizar análises mais detalhadas no espaço geográfico contaminado e sua evolução temporal, incluindo uma classificação qualitativa de risco que considera os sistemas complexos de múltiplas rotas de exposição e contaminantes.

A avaliação de risco, realizada com o modelo SysRisk, apresenta resultados quantitativa e qualitativamente diferentes dos obtidos na abordagem utilizada no trabalho de Lima (2009) para o mesmo subconjunto de dados de campo das concentrações de contaminantes.

Os resultados do SysRisk ofereceram um nível de informação mais detalhado na construção dos indicadores para a caracterização do risco. A análise espaçotemporal de $\mathrm{HI}$ e CR mostrou ser útil para subsidiar, de forma mais eficiente, a elaboração dos planos de mitigação de riscos diferenciando subáreas e subperíodos do cenário contaminado.

A proposta da Máquina de Vetores Suporte, como técnica de classificação em categorias de risco, mostrou-se eficiente, indicando que pode ser uma boa alternativa para a análise dos riscos da exposição a múltiplos contaminantes simultâneos nos diferentes elementos da matriz espaço-temporal proposta.

A abordagem qualitativa, que pode ter uma maior facilidade na comunicação e compreensão dos riscos pela população, pode permitir, assim, ações sociais considerando também a importância dos efeitos da exposição simultânea a vários contaminantes.

Por outro lado, a caracterização de risco, com as classes categóricas de risco, permite diminuir custos com diversos processos avaliativos e embasar adequadamente o planejamento e programas de gerenciamento de riscos visando a gestão ou recuperação de áreas afetadas.

A re-análise parcial dos riscos, realizada no presente trabalho, acrescentou alguns resultados de indicadores de risco importantes que poderão ser incluídos nos planos de gestão de risco para o caso do descomissionamento da metalúrgica de zinco estudada, em particular pela exposição ao cobalto, que teve um tratamento diferenciado neste trabalho.

\section{REFERÊNCIAS}

ANDERSON, D. R.; SWEENEY D. J.; WILLIAM T. A. Estatística Aplicada à administração e economia. São Paulo: Editora Cengage Learning, 2a. ed., 2007.

ATSDR - Agency for Toxic Substances and Disease Registry. Public Health Assessment Guidance Manual, Atlanta, Georgia, 2005.

ATSDR - Agency for Toxic Substances and Disease Registry. Disp. em: http://www.atsdr.cdc.gov/. Acessado em: outubro/ 2015.

BAES, C. F.; SHARP, R. D; SJORJEEN, A. L.; SHOR, R.W. Review and analysis of parametres and assessing transport of environmentally released radionuclides through agriculture. Oak Ridge National Laboratory. Oak Ridge Tennessee, 1984.

BARNES, D. G.; DOURSON, M.; PREUSS, P.; BELLIN, J.; DEROSA, C.; ENGLER, R.; GHALI, G. Reference dose (RfD): Description and use in health risk assessments. Regulatory Toxicology and Pharmacology, v. 8, n. 4, p. 471-486, 1988.

BRILHANTE O. M. \& CALDAS, L. Q. A. Gestão de risco em saúde ambiental. Rio de Janeiro: FIOCRUZ, 158p., 2004.

CAO Q.; YU Q.; CONNELL, D. W. Health risk characterisation for environmental pollutants with a new concept of overall risk probability, Journal of Hazardous Materials, v. 187, n. 1-3, p. 480-487, 2011.

CETESB - Companhia Ambiental do Estado de São Paulo.
Evaluation of toxicological risk to human health. Manual de Gerenciamento de Áreas Contaminadas. Cetesb-GTZ Project, Chapter's VIII, IX. São Paulo, 2001.

CETESB - Companhia Ambiental do Estado de São Paulo. Norma Técnica P4.261. Maio/2003.

CETESB - Companhia Ambiental do Estado de São Paulo. Gerenciamento de Risco. http://www.cetesb.sp.gov.br /gerenciamento-de-riscos/ Emergências-Químicas/17-Referencias. Acessado em: março/2015.

CETESB - Companhia Ambiental do Estado de São Paulo. ANA - Agência Nacional de Águas. Guia Nacional de Coleta e Preservação de Amostras: água, sedimento, comunidades aquáticas e efluentes líquidos. Brasília, 2011. DOURSON, M. L; ANDERSEN, M. E.; ERDREICH, L. S.; MACGREGOR, J. A. Using human data to protect the public's health. Regulatory Toxicology and Pharmacology, v. 33, n. 2, p. 234-256, 2001.

EDLER, L.; POIRIER, K.; DOURSON, M.; KLEINER, J.; MILESON, B.; NORDMANN, H.; RENWICK, A.; SLOB, W.; WALTON, K.; WÜRTZEN, G.; Mathematical modelling and quantitative methods. Food and Chemical Toxicology, v. 40, n. 2, p. 283-326, 2002.

FACELI, K.; LORENA, A. C.; GAMA, J.; CARVALHO, A. C. P. L. F. Inteligência Artificial - Uma Abordagem de Aprendizado de Máquina. Rio de Janeiro: LTC Genio, 2011. 
FUNDACENTRO/MINISTÉRIO DO TRABAHO E EMPREGO. Avaliação Qualitativa de Riscos Químicos: orientações básicas para controle da exposição a produtos químicos. São Paulo, 2012.

GOLDER. Guidance document for country foods surveys for the purpose of human health risk assessment. Submitted to: Health Canada. Golder Associates Ltd., Canadá, 2005.

GUO, G.; ZHANG C.; WU G.; DING Q.; WANG S.; LI F. Health and ecological risk-based characterization of soil an sediment contamination in shipyard with long-term use of DDT-containing antifouling paint. Science of the Total Environment, v. 450-451, p. 223-229, 2013.

HALL, M.; FRANK, E.; HOLMES, G.; PFAHRINGER, B.; REUTEMANN, P.; WITTEN, I. H. O software WEKA Data Mining: Uma Atualização. ACM SIGKDD explorations newsletter. v. 11, n. 1, p. 10-18, 2009.

HANLEY, J. A. \& MCNEIL, B. J. The meaning and use of the area under a receiver operating characteristic (ROC) curve. Radiology, v. 143, n. 1, p. 29-36, 1982.

HEALTH CANADA. Canadian Handbook on Health Impact Assessment. V. 1, n. 3: The Multidisciplinary Team. Ottawa, ON. Canada, 2004.

HEALTH CANADA. Federal Contaminated Site Risk Assessment in Canada. Part I: Guidance on Human Health Preliminary Quantitative Risk Assessment (PQRA). 2012.

HSU, C. W. \& LIN, C. J. A comparison of methods for multiclass support vector machines. Neural Networks, IEEE Transactions on, v. 13, n. 2, p. 415-425, 2002.

IARC - International Agency for Research on Cancer - WHO. List of Classifications. Agents Classified by the IARC Monographs, v. 1 - 109. Disp. em: http://monographs.iarc.fr/ ENG/Classification/index.php. Acessado em: abril/2015.

LIMA, C. A. Avaliação de Risco Ambiental como Ferramenta para o Descomissionamento de uma Indústria de Metalurgia de Zinco. Rio de Janeiro, 2009. 131 p. Tese (Doutorado), Universidade Federal do Rio de Janeiro.

LINKOV, I.; LONEY, D.; CORMIER, S.; SATTERSTROM, F. K.; BRIDGES, T. Weight-of-evidence evaluation in environmental assessment: review of qualitative and quantitative approaches. Science of the Total Environment, v. 407, n. 19, p. 5199-5205, 2009.

MARHAVILAS, P. K.; KOULOURIOTIS, D.; GEMENI, V. Risk analysis and assessment methodologies in the work sites: On a review, classification and comparative study of the scientific literature of the period 2000-2009. Journal of Loss Prevention in the Process Industries, v. 24, n. 5, p. 477-523, 2011.

MENZIE, C. A. \& FRESHMAN, J. S. An assessment of the risk assessment paradigm for ecological risk assessment. Human and Ecological Risk Assessment, v. 3, n. 5, p. 853-892, 1997.

NRC - National Research Council. Risk assessment in the Federal Government: managing the process. Washington, DC: National Academy Press, 1983.

PLATT, J. Sequential minimal optimization: A fast algorithm for training support vector machines. 1998.

PNUMA - Programa de las Naciones Unidas para el Medio Ambiente, IPCS, Evaluación de riesgos químicos: evaluación de riesgos humanos, evaluación de riesgos ambientales evaluación de riesgos ecológicos. Módulo de capacitación nº. 3, PNUMA/IPCS, 1999.

RENWICK, A. G.; BARLOW, S. M.; HERTZ-PICCIOTTO, I.; BOOBIS, A. R.; DYBING, E.; EDLER, L.; EISENBRAND, G.; GREIG, J. B.; KLEINER, J.; LAMBE, J.; MULLER, D. J. G.; SMITH, M. R.; TRITSCHER, A.; TUIJTELAARS, S.; VAN
DEN BRANDT, P. A.; WALKER, R.; KROES, R. Risk characterisation of chemicals in food and diet. Food and Chemical Toxicology, v. 41, n. 9, p. 1211-1271, 2003.

RIFKIN, R. \& KLAUTAU, A. In defense of one-vs-all classification. The Journal of Machine Learning Research, v. 5, p. 101-141, 2004

RIVM - National Institute for Public Health and the Environment. PROAST: Software for dose-response modeling and benchmark dose analysis. http://www.rivm. nl/en/foodnutritionandwater/foodsafety/proast.jsp. 2009.

SOUZA, F. M. N.; SILVA, C. E.; AGUIAR, L. A.; ALMEIDA, J. R. Análise de riscos como instrumento para sistemas de gestão ambiental. Aquidabã, Revista Ibero-Americana de Ciências Ambientais, v.3, n.1, p.17- 41, 2012.

UE-IHCP, US Ropean Commission-Joint Research Center. Techinical Guidance Document - Part I. USR20418 EN/1, Directive 98/8EC. 2003.

USEPA - United States Environmental Protection Agency. Guidelines for Carcinogen Risk Assessment. EPA/630/R00/004. Washington (DC), USA, 1986.

USEPA - United States Environmental Protection Agency. Risk Assessment Guidance for Superfund. Vol I: Human Health Evaluation Manual (Part A). Washington - DC, 1989.

USEPA - United States Environmental Protection Agency. Guidelines for Exposure Assessment. EPA/6--/Z-92/001. 1992.

USEPA - United States Environmental Protection Agency. Exposure Factors Handbook. USEPA - Region III. August, 1997.

USEPA - United States Environmental Protection Agency. Science Policy Council Handbook. EPA-100-B-00-002. 2000.

USEPA - United States Environmental Protection Agency. Risk Assessment Guidance for Superfund. Vol I: Human Health Evaluation Manual (Part E, Supplemental Guidance for Dermal Risk Assessment). USA, Washington, DC. 2004(a)

USEPA - United States Environmental Protection Agency. Example Exposure Scenarios. USA, Washington, DC, 2004(b).

USEPA - United States Environmental Protection Agency. Consolidated List of Chemicals Subject to the Emergency Planning and Community Right-to-Know Act (EPCRA) Section 304. 2014.

USEPA - United States Environmental Protection Agency/ IRIS-Integrated Risk Information System. Disp. em: http://www.epa.gov/IRIS/. Acessado em: abril/2015.

VAPNIK, V. A; BOSER, B.; GUYON, M. Training Algorithm for Optimal Margin Classifiers. Computational Learning Theory. P. 144-152, 1992.

WESTON, J. \& WATKINS, C. Multi-class Support Vector Machines - Technical Report, CSD-TR-09-04, University of London, Department of Computer Science. 1998.

WHO-IPCS - World Health Organization - International Programme on Chamical Safety. Risk Assessment Toolkit: chemical hazards. USA. 2010.

WHO - World Health Organization. International Programme on Chamical Safety - ICHEM. Disp. em: http://www.inchem. org/; Acessado em: abril/2015.

WOOD, J. M. Understanding and Computing Cohen’s Kappa: A Tutorial. WebPsychEmpiricist. 2007.

XU, J. An extended one-versus-rest support vector machine for multi-label classification. Neurocomputing, v. 74, n. 17, p. 3114-3124, 2011.

Submetido em 16 de outubro de 2016 Aceito em $1^{\circ}$ de março de 2017 